\title{
Unveiling the Dynamical State of Massive Clusters through the ICL Fraction
}

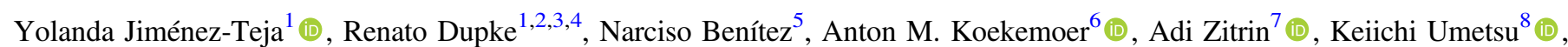
Bodo L. Ziegler ${ }^{9}$, Brenda L. Frye ${ }^{10}$, Holland Ford ${ }^{11}$ (1) Rychard J. Bouwens ${ }^{12}$ (1) , Larry D. Bradley ${ }^{6}$ (1) , Thomas Broadhurst $^{13,14}$, Dan Coe ${ }^{6}$ (1), Megan Donahue ${ }^{15}$ (10), Genevieve J. Graves ${ }^{16}$, Claudio Grillo ${ }^{17}$, Leopoldo Infante ${ }^{18}$ (1), Stephanie Jouvel ${ }^{19}$, Daniel D. Kelson ${ }^{20}$ (1), Ofer Lahav ${ }^{21}$, Ruth Lazkoz ${ }^{13}$, Dorom Lemze ${ }^{11}$, Dan Maoz ${ }^{22}$, Elinor Medezinski ${ }^{23}$ (1) , Peter Melchior ${ }^{23}$ (1), Massimo Meneghetti ${ }^{24,25}$ (1), Amata Mercurio ${ }^{26}$, Julian Merten ${ }^{27}$, Alberto Molino ${ }^{5,28}$ (1) , Leonidas A. Moustakas ${ }^{29}$ (1), Mario Nonino ${ }^{30}$, Sara Ogaz ${ }^{11}$, Adam G. Riess ${ }^{6,11}$, Piero Rosati ${ }^{31}$, Jack Sayers ${ }^{32}$ (1), Stella Seitz ${ }^{33,34}$ (1) , and Wei Zheng ${ }^{11}$ (1) 1 Observatório Nacional, Rua General José Cristino, 77-Bairro Imperial de São Cristóvão, Rio de Janeiro, 20921-400, Brazil; yojite@iaa.es, yolanda@on.br
2 Department of Physics and Astronomy, University of Alabama, Box 870324, Tuscaloosa, AL 35487, USA

${ }^{3}$ Department of Astronomy, University of Michigan, 311 West Hall, 1085 South University Ave., Ann Arbor, MI 48109-1107, USA ${ }^{4}$ Eureka Scientific Inc., 2452 Delmer St., Suite 100, Oakland, CA 94602, USA

5 Instituto de Astrofísica de Andalucía (CSIC), Glorieta de la Astronomía s/n, Granada, E-18008, Spain

${ }^{6}$ Space Telescope Science Institute, 3700 San Martin Dr., Baltimore, MD 21218, USA

7 Physics Department, Ben-Gurion University of the Negev, P.O. Box 653, Be'er-Sheva 8410501, Israel

${ }_{9}^{8}$ Institute of Astronomy and Astrophysics, Academia Sinica, P.O. Box 23-141, Taipei 10617, Taiwan

9 Department of Astrophysics, University of Vienna, Türkenschanzstrasse 17, A-1180 Vienna, Austria

${ }^{10}$ Department of Astronomy, Steward Observatory, University of Arizona, 933 North Cherry Avenue, Tucson, AZ 85721, USA

11 Department of Physics and Astronomy, The Johns Hopkins University Homewood Campus, Baltimore, MD 21218, USA ${ }^{12}$ Leiden Observatory, Leiden University, NL-2300 RA Leiden, The Netherlands

${ }^{13}$ Fisika Teorikoa, Zientzia eta Teknologia Fakultatea, Euskal Herriko Unibertsitatea UPV/EHU, E-48080 Bilbao, Spain

${ }_{15}^{14}$ IKERBASQUE, Basque Foundation for Science, Alameda Urquijo, 36-5, E-48008 Bilbao, Spain

${ }^{15}$ Physics and Astronomy Department, Michigan State University, East Lansing, MI 48824 USA ${ }^{16}$ Department of Astronomy, University of California, Berkeley, CA 94720, USA

${ }^{17}$ Dark Cosmology Centre, Niels Bohr Institute, University of Copenhagen, Juliane Maries Vej 30, DK-2100 Copenhagen, Denmark

${ }^{18}$ Instituto de Astrofísica y Centro de Astroingeniería, Facultad de Física, Pontificia Universidad Católica de Chile,

Vicuña Mackenna 4860, 7820436 Macul, Santiago, Chile
19 Department of Physics and Astronomy, University College London, 132 Hampstead Road, London NW1 2PS, UK

${ }^{20}$ The Observatories of the Carnegie Institution for Science, 813 Santa Barbara St., Pasadena, CA 91101, USA

${ }^{21}$ Department of Physics and Astronomy, University College of London, Gower Street, London WC1E 6BT, UK ${ }^{22}$ School of Physics and Astronomy, Tel-Aviv University, Tel-Aviv 6997801, Israel

23 Department of Astrophysical Sciences, Princeton University, Princeton, NJ 08544, USA ${ }^{24}$ Osservatorio Astronomico di Bologna, Via Gobetti 93/3, I-40129, Bologna, Italy

25 INFN, Sezione di Bologna, viale Berti Pichat 6/2, I-40127 Bologna, Italy

${ }^{26}$ INAF-Osservatorio Astronomico di Capodimonte, Salita Moiariello 16 I-80131 Napoli, Italy

${ }^{28}$ Instituto de Astronomia, Geofísica e Ciências Atmosféricas, Universidade de São Paulo, 05508-090 São Paulo, Brazil

29 Jet Propulsion Laboratory, California Institute of Technology, MS 169-506, 4800 Oak Grove Drive, Pasadena, CA 91109, USA

${ }^{30}$ INAF-Osservatorio Astronomico di Trieste, via G. B. Tiepolo 11, I-34143 Trieste, Italy

${ }^{31}$ Dipartimento di Fisica e Scienze della Terra, Università degli Studi di Ferrara, Via Saragat 1, I-44122 Ferrara, Italy

${ }^{32}$ Division of Physics, Math, and Astronomy, California Institute of Technology, Pasadena, CA 91125, USA

${ }^{33}$ University Observatory Munich, Scheinerstrasse 1, D-81679 Munich, Germany

${ }^{34}$ Max Planck Institute for Extraterrestrial Physics, Giessenbachstrasse, D-85748 Garching, Germany

Received 2018 January 31; revised 2018 March 6; accepted 2018 March 13; published 2018 April 17

\begin{abstract}
We have selected a sample of 11 massive clusters of galaxies observed by the Hubble Space Telescope in order to study the impact of the dynamical state on the intracluster light (ICL) fraction, the ratio of total integrated ICL to the total galaxy member light. With the exception of the Bullet cluster, the sample is drawn from the Cluster Lensing and Supernova Survey and the Frontier Fields program, containing five relaxed and six merging clusters. The ICL fraction is calculated in three optical filters using the CHEFs ICL estimator, a robust and accurate algorithm free of a priori assumptions. We find that the ICL fraction in the three bands is, on average, higher for the merging clusters, ranging between $\sim 7 \%$ and $23 \%$, compared with the $\sim 2 \%-11 \%$ found for the relaxed systems. We observe a nearly constant value (within the error bars) in the ICL fraction of the regular clusters at the three wavelengths considered, which would indicate that the colors of the ICL and the cluster galaxies are, on average, coincident and, thus, so are their stellar populations. However, we find a higher ICL fraction in the F606W filter for the merging clusters, consistent with an excess of lower-metallicity/younger stars in the ICL, which could have migrated violently from the outskirts of the infalling galaxies during the merger event.
\end{abstract}

Key words: galaxies: clusters: general - techniques: image processing

\section{Introduction}

The intracluster light (ICL) is starting to get some attention for its ability to give insights into the processes driving galaxy cluster evolution. Defined as the light of the stars that do not belong to any of the galaxies of the clusters but are gravitationally bound to the potential of the system, the ICL origin, general properties, and evolution are almost completely unknown. Its contribution to the total luminosity of the cluster can be significant, ranging from $10 \%$ to $50 \%$ of the total light, where the upper limit was claimed by Bernstein et al. (1995) in 
the core of the Coma cluster. This contribution is known as the ICL luminosity fraction (hereafter the ICL fraction), and it is formally defined as the ratio between the ICL and the total luminosity of the cluster. The total luminosity comprises the ICL and the light from the galaxy members of the cluster. Although this parameter is conceptually very simple, accurate measurements of the ICL are not trivial to obtain, which largely explains the scatter in the results reported in different studies in the literature with inconsistent methodologies. Disentangling the ICL from the light of the stars in the galaxies is not straightforward, being especially complex in the case of the brightest cluster galaxy (BCG). Traditional methods are often ambiguous, relying on different a priori hypotheses that tie conditions to the final measurements, leading to different conclusions on the nature of the ICL and its properties. Assuming that the ICL formation is mainly driven by ongoing processes (e.g., tidal stripping or total disruption of dwarf galaxies), it is predicted that the ICL fraction will grow with decreasing redshift (Willman et al. 2004; Rudick et al. 2006, 2011; Krick \& Bernstein 2007; Burke et al. 2012). However, several works failed to find any significant redshift dependence of the ICL or the ICL fraction probed at different redshift ranges (Krick \& Bernstein 2007; Guennou et al. 2012; Montes \& Trujillo 2018; Morishita et al. 2017). In addition, it has been suggested that the ICL fraction should be related to the dynamical evolutionary stage of the cluster, such as a merger, since it is expected that the amount of ICL would increase with the infall material on the cluster (Adami et al. 2004, 2013; Pierini et al. 2008). In fact, although involving a smaller scale, some authors reported a correlation between the fraction of diffuse light and the dynamical state in groups, finding that the intragroup light (IGL) fraction was higher in the case of active groups (e.g., Da Rocha \& Mendes de Oliveira 2005; Da Rocha et al. 2008).

The major difficulty in looking for quantitative and qualitative relations between the ICL and other cluster properties comes from a combination of small-number statistics of clusters with measured ICL, the different data quality, and the use of very disparate methodology. In particular, the latter makes direct comparisons of results from different works extremely difficult, since we cannot determine if the conclusions are in fact real or the result of a bias induced by the different techniques used, the different hypotheses assumed, or selection effects. The aim of this work is to study in a consistent way the role of the cluster's dynamics in the ICL formation. We selected a significant sample of massive merging and relaxed clusters spanning a redshift range of $0.18<z<0.54$, which is of special interest, since several works suggest that the ICL is mainly formed at later times, i.e., $z \leqslant 1$ (e.g., Burke et al. 2012; Montes \& Trujillo 2014, 2018; Morishita et al. 2017), with the most dramatic evolution in the ICL fraction happening at $z \sim 0.5$ (Montes \& Trujillo 2018). We estimated the ICL fraction in this sample using an accurate technique free of a priori assumptions called the CHEFs ICL Estimator (CICLE; Jiménez-Teja \& Dupke 2016). CICLE is based on the use of Chebyshev-Fourier functions (CHEFs; Jiménez-Teja \& Benítez 2012) to model the surface light distribution of the galaxies and curvature maps to disentangle the ICL from the light of the BCG. CICLE studies the ICL twodimensionally, without the need to simplify its surface distribution to a profile and, thus, not assuming any kind of symmetric distribution. It does not assume any previous hypothesis either, apart from the fact that the radial profiles of the BCG and the ICL must be different, i.e., analogous to having different "slopes," which in practice is translated in surface analysis as having different curvatures. Notice that this is a minimum condition, since if it is violated, no method would be able to disentangle the two light distributions. CICLE has been successfully tested with mock data and applied to real data from cluster Abell 2744 (Jiménez-Teja \& Dupke 2016).

The test sample used in this work consists of 11 massive clusters with well-defined dynamical states and similar observational characteristics, all with Hubble Space Telescope (HST) observations available. Most of the clusters are part of the Cluster Lensing and Supernovae Survey with Hubble (CLASH; Postman et al. 2012) and the Frontier Fields (FF) program (Lotz et al. 2017). Both programs provide data of exceptional quality and depth, ideal for using CICLE. In this work, we present the analysis of the ICL fraction for these 11 massive clusters in three different $H S T$ bands. We divided the sample into two groups, merging and relaxed clusters, aiming to study their ICL fraction colors (defined as the difference between two measurements of the ICL fraction of a cluster made at different wavelengths) and to unveil the possible progenitors of this ICL. The paper is organized as follows. Section 2 describes the clusters in our sample and the criteria used to choose them. The observational characteristics of the data used and the preprocessing carried out are explained in Section 3, while the CICLE algorithm is outlined in Section 4. In Section 5, we describe previous results regarding the ICL of the clusters in our sample in order to compare them, to the extent possible, with the results that we obtain with CICLE in Section 6. Finally, we discuss our results in Section 7 and draw the conclusions in Section 8. Throughout the paper, we assume a standard $\Lambda \mathrm{CDM}$ cosmology with $H_{0}=70 \mathrm{~km} \mathrm{~s}^{-1}$, $\Omega_{m}=0.3$, and $\Omega_{\Lambda}=0.7$.

\section{Sample Selection}

The data used for this work come mainly from CLASH ${ }^{35}$ (Postman et al. 2012) and the FF program ${ }^{36}$ (Lotz et al. 2017), which includes both relaxed and merging massive clusters observed by the HST.

The CLASH program was mainly devoted to studying the dark matter distribution in galaxy clusters using both strong and weak lensing and to search for SNe Ia out to redshift $z \sim 2$ in 25 specially selected massive clusters. Twenty of these 25 systems were initially chosen to be likely relaxed, according to their symmetric surface brightness distribution in the X-ray. The remaining five clusters were selected for being well-known high-magnification lenses. The cluster sample is distributed in the range $0.15<z<0.9$ and has masses in the range $5<M_{\mathrm{vir}}<30 \times 10^{14} M_{\odot}$ (Postman et al. 2012; Umetsu et al. 2014; Merten et al. 2015). All of them have X-ray temperatures $T_{X}>5 \mathrm{keV}$. Each cluster was observed with both the Advanced Camera for Surveys (ACS) and the Wide Field Camera 3 (WFC3) in 16 passbands covering the NUV, optical, and NIR wavelengths (Postman et al. 2012).

The FF program (PI: Lotz) has also observed with unprecedented depth six massive clusters using both ACS and WFC3. These systems were also chosen for being wellknown high-magnification lenses, with the aim of not only

\footnotetext{
35 http://www.stsci.edu/ postman/CLASH/Home.html

36 http://www.stsci.edu/hst/campaigns/frontier-fields/FF-Data
} 
studying the dark matter distribution in their cores but also analyzing the distant galaxies in the background, improving our knowledge of the universe in the epoch of reionization. The six clusters, with redshifts ranging from $z \sim 0.3$ to $\sim 0.55$ and masses spanning the interval $\sim 10<M_{\mathrm{vir}}<30 \times 10^{14} M_{\odot}$, have been observed in seven different optical and NIR bands using 840 Hubble orbits (Lotz et al. 2017).

Our sample is composed of six CLASH clusters (Abell 383, Abell 611, MS 2137-2353, MACS 1115.9+0129, RXJ 2129.7 +0005 , and Abell 209), four FF clusters (Abell 2744, MACS J0416.1-2403, MACS J0717.5+3745, and MACS J1149.5 +2223 ), and the western subcluster of the Bullet system. Although MACS J0416.1-2403, MACS J0717.5+3745, and MACS J1149.5+2223 also belong to the CLASH sample, we used the FF images to analyze them, given that the CLASH data are included in them. Two more clusters were initially considered-the eastern subcluster in Bullet and the CLASH cluster MACS 1931.8-2635-but, due to the pollution from a nearby bright star in the first case and the lack of enough spectra in the HST field of view in the second, these two systems were not included in the final study. The criteria to select these systems were (a) having similar masses, (b) having high-quality $H S T$ data available, (c) having enough spectroscopic information available on the galaxies in the images for the cluster membership determination, and (d) having a welldefined dynamical state, as determined by several indicators, if possible. The goal is to consistently study the ICL fraction with respect to the dynamical stage of the systems, using a homogeneous sample of objects under the same observational characteristics with deep-imaging data and a significant number of precise galaxy redshifts. Splitting the sample according to the dynamical stage is thus crucial to disentangling which are the main mechanisms responsible for the ICL formation in each case.

The dynamical state of a cluster has been traditionally determined through visual inspection analyzing the morphology and the presence of substructure. Regular (relaxed) systems are, by definition, virialized, so they should be roughly circular, symmetric, and without tidal features. They usually exhibit higher concentration indexes $c$ (here defined as the ratio between the light enclosed by a fixed inner aperture and the total light of the cluster) than unrelaxed systems (Cassano et al. 2010; Donahue et al. 2016). The existence of multiple BCGs is also an indicator of dynamical activity in a cluster related to its appearance. Furthermore, we also considered other parameters measured in X-rays, such as the symmetry of the gas distribution. Dynamical interactions produce shocks or pressure waves that often break the symmetry of the gas distribution. Deviations from this symmetry are quantified through the power ratio and the axial ratio. The power ratio is a multipole decomposition of the $\mathrm{X}$-ray surface brightness distribution that is sensitive to the presence of substructure, while the axial ratio is simply the ratio between the lengths of the minor and major axes of the X-ray distribution, thus providing an idea of its degree of elongation (see Cassano et al. 2010 and Donahue et al. 2016 for a detailed description of these parameters). Also, the centroid shift $w$, defined as a statistical measurement of the projected offset between the X-ray peak and the centroid of the cluster measured within different circular apertures, serves to quantify the dynamical state of the system. It is expected that in relaxed clusters, the gravitational potential dominates the geometry of the system, making the hot X-ray-emitting gas approximately align with the total mass distribution.

In the last decades, the presence of radio halos and relics has also been associated with merging clusters (e.g., Cassano et al. 2010, 2016; Cuciti et al. 2015; Pandey-Pommier et al. 2016), especially in the case of massive systems, through diffusive shock acceleration (e.g., Enßlin et al. 1998). Electrons in the intracluster gas are accelerated diffusively, using part of the energy dissipated during mergers in active clusters to energies where they would emit cluster-scale ( $\sim \mathrm{Mpc}$-scale) synchrotron radiation. This emission appears in nonrelaxed clusters in the form of giant radio halos or giant radio relics. Relatively passive systems with cool cores also exhibit diffuse synchrotron radio emission, but on smaller scales $(\sim 100-300 \mathrm{kpc})$, in what is known as radio mini-halos. In this case, the electron acceleration process is likely produced by active galactic nuclei (AGN)-driven turbulence in cool-core clusters (i.e., clusters with temperature profiles falling toward the center) causing this nonthermal emission (Brunetti \& Jones 2014) and is not related to the overall cluster dynamical state (e.g., Bravi et al. 2016).

Before describing the sample, it is important to keep in mind that it is much easier to tell if the cluster is merging than if it is relaxed. If there is no evidence of departures from relaxation, we consider the clusters to be relaxed. One should notice that the results of the analysis will not be dependent on the precise estimation of the relaxation level, just that we compare clusters that have plenty of evidence of merging with those that do not. We will now describe the properties of each one of the 11 clusters in our sample, particularly their dynamical states, according to all of the indicators previously described.

1. Abell 383 (hereafter A383; $z \sim 0.187$ ) is identified as a relaxed cluster according to the X-ray morphological parameter diagrams built by Cassano et al. (2010; see Figure 1 in Cassano et al. 2010 and Figure 3 in Donahue et al. 2016). Its power ratios are very small, and its X-ray distribution is highly circular, with an axis ratio of $\sim 0.97 \pm 0.01$ within a metric radius of $500 \mathrm{kpc}$ (Donahue et al. 2016). It has a cool core with just a single point radio source of less than $5 \mathrm{kpc}$ detected at the BCG (Giacintucci et al. 2017). All evidence suggests that this is a regular system.

2. Abell 611 (hereafter A611; $z \sim 0.288$ ) is also part of the CLASH sample displaying a circular and symmetric distribution in the X-ray (Postman et al. 2012). Although bright in the $\mathrm{X}$-ray, it is clearly relaxed, as the measurements of X-ray concentration, centroid shift, and power ratios made by Donahue et al. (2016) indicate. No diffuse extended radio emission is detected, just central emission connected to the BCG (Venturi et al. 2008; Pandey-Pommier et al. 2016).

3. MS 2137-2353 (hereafter MS 2137; $z \sim 0.313$ ) appears to be a well-relaxed cluster, as its X-ray morphological measurements suggest (Donahue et al. 2016). We did not find any information on possible radio emission available in the literature.

4. MACS $1115.9+0129$ (hereafter MACS $1115 ; z \sim 0.352$ ) is a cool-core cluster that is not as circular in the $\mathrm{X}$-ray as other clusters in the CLASH sample (AR $\sim 0.85 \pm 0.03$ ) but has a high X-ray concentration and small centroid shift and power ratios (Donahue et al. 2016), all compatible with a relaxed state. A radio mini-halo was 
detected by Kale et al. (2013, 2015), Pandey-Pommier et al. (2016), and Giacintucci et al. (2017).

5. RXJ 2129.7+0005 (hereafter RXJ 2129; $z \sim 0.234$ ) is also a clearly relaxed cluster according to its X-ray morphological properties, although its axis ratio is not as high as that from other systems in the CLASH "relaxed" sample ( $\mathrm{AR} \sim 0.87 \pm 0.01$; Donahue et al. 2016). It has a cool core. Its BCG hosts a strong radio source that is surrounded by a mini-halo (Kale et al. 2015; PandeyPommier et al. 2016; Giacintucci et al. 2017).

6. Abell 209 (hereafter A209; $z \sim 0.206$ ) is a rich, X-rayluminous cluster (Mercurio et al. 2003a, 2003b) that was originally selected as a relaxed cluster in the CLASH survey due to its symmetric X-ray distribution, although several indications of marginal departures from relaxation were also pointed out (Postman et al. 2012). It is a noncool-core cluster, showing substructure in the galaxy velocity distribution and a marked luminosity segregation, strongly suggesting a merging state (Mercurio et al. 2003a, 2003b). Despite having a small centroid shift and power ratios, its X-ray concentration is compatible with that of a merging cluster (Donahue et al. 2016). The presence of a giant radio halo associated with its BCG (Venturi et al. 2007; Pandey-Pommier et al. 2016; Giacintucci et al. 2017) seems to confirm that A209 is in fact a nonrelaxed cluster, and it is either undergoing a merging event or at the end of a massive merger phase (Venturi et al. 2007; Pandey-Pommier et al. 2016).

7. Abell 2744 (hereafter A2744; $z \sim 0.307$ ) is the first cluster observed by the FF program. It is a richness 3 cluster with a significant enhancement of the blue galaxy population (a blue fraction $\sim 2.2 \pm 0.3$ higher than that found in the same core regions of nearby clusters; Couch \& Sharples 1987) mainly composed of starburst and poststarburst galaxies (Couch 1998). Analyzing combined X-ray and spectroscopic data, Owers et al. (2011) identified two major substructures in the velocity distribution corresponding to the remnants of two major subclusters in a post-core-passage phase of merging with a large line-of-sight component, along with an interloping minor merger model. This model was later confirmed and refined by Merten et al. (2011), who concluded that it is in fact a quadruple merging system. This result confirmed the previous works addressing the unrelaxed dynamical state of A2744 by Kempner \& David (2004) and Boschin et al. (2006). It hosts a giant radio halo first found by Giovannini et al. (1999) and later confirmed by Venturi et al. (2008), Kale et al. (2013), and Giacintucci et al. (2017), as well as a single radio relic in the outskirts (Govoni et al. 2001a, 2001b; Kale et al. 2015). All of the evidence points to a heavily disturbed merging system.

8. MACS J0416.1-2403 (hereafter MACS 0416; $z \sim 0.396$ ) is the most elongated cluster in the CLASH sample. It is a high-magnification gravitational lens with an associated ultra-steep-spectrum radio (USSR) halo. It is the most powerful halo ever observed (Pandey-Pommier et al. 2016). Its power ratios, significantly higher than the average of the CLASH relaxed sample, also suggest a nonvirialized state. With a low X-ray concentration and high centroid offset, it is clearly classified as a nonrelaxed cluster according to the Cassano et al. (2010) diagram (Donahue et al. 2016). Moreover, there are several shifts between the peaks of lensing mass and the X-ray and radio emission, which, in conjunction with the presence of the USSR halo, point to an impressive four-cluster post-merging scenario similar to that of A2744, the Pandora cluster (Pandey-Pommier et al. 2016).

9. MACS J0717.5+3745 (hereafter MACS 0717; $z \sim$ 0.548) is the CLASH system with the lowest X-ray concentration and highest centroid shift, also showing high dipole power ratios (Donahue et al. 2016). It hosts a very powerful radio halo and a bright relic located between the merging structures of the cluster (Van Weeren et al. 2009; Pandey-Pommier et al. 2016). An offset between the mass, X-ray, and radio distribution peaks is observed, which suggests that the system is an ongoing merger in this case, since the steepness of the hosted radio halo is lower than that of MACS 0416 (Pandey-Pommier et al. 2016).

10. MACS J1149.5+2223 (hereafter MACS 1149; $z \sim$ $0.544)$ is the MACS cluster with the highest velocity dispersion $\left(\sim 1800 \mathrm{~km} \mathrm{~s}^{-1}\right)$. According to X-ray morphological diagrams by Cassano et al. (2010) and measurements by Donahue et al. (2016), this system is clearly classified as merging. The X-ray analysis performed by Ogrean et al. (2016) confirms that this is a merging system with several substructures, displaying a line-of sight component, and with no evidence of a compact cool core. However, the lack of temperature substructures or surface brightness features, which would be expected in such a complex merger, suggests that MACS 1149 is an old merger. This scenario is supported by Bonafede et al. (2012), who reported evidence for a giant, very steep radio halo, as well as a double relic system, which could point to a post-violent merger phase (Pandey-Pommier et al. 2016). In addition, the dynamical analysis by Golovich et al. (2016) confirms that this system seems to be composed of two different mergers involving three subclusters.

11. The cluster 1E 0657-558 (hereafter Bullet; $z \sim 0.296$ ) has been thoroughly studied in the literature since its discovery in the 1990s (e.g., Barrena et al. 2002 and references thereafter). It is a textbook case for merging clusters, and its nickname comes from the prominent Mach cone observed in X-rays, originated by a merger between two clusters very close to the plane of the sky (Markevitch et al. 2002, 2004). It is an ongoing merger where an infalling subcluster is observed just after its first core passage (Springel \& Farrar 2007). The presence of a radio halo was first noticed by Liang et al. (2000), and several other authors have confirmed this detection at different levels of significance (e.g., Shimwell et al. 2014).

\section{Data}

We analyzed the HST ACS images obtained in the CLASH and FF programs. The formidable depth and quality of the HST data are fundamental to studying the ICL, given its low surface brightness of, typically, $\mu_{V} \geqslant 26.5 \mathrm{mag} \operatorname{arcsec}^{2}$ (e.g., Montes $\&$ Trujillo 2014). Deep ground-based data could also be used to detect and measure the ICL, although the fact that the absolute level of background contribution is larger makes the use of $H S T$ data preferable to decrease the uncertainty introduced by 
this component. Due to the reduced field of view of the ACS / WFC instrument $\left(202 \times 202 \operatorname{arcsec}^{2}\right)$, some clusters had effectively imaged only its central area. CLASH data are available in eight optical ACS filters: F435W, F475W, F555W, F606W, F625W, F775W, F814W, and F850LP, whereas the FF images are observed in just three of them: F435W, F606W, and F814W. We decided to analyze the three common bands, not only to compare the ICL fractions between merging and relaxed clusters at the same wavelength but also to study if any statistical trends in ICL fraction colors could be identified. Detailed analysis of stellar populations in the ICL is beyond the scope of this paper and will be covered in a future paper, and that is why we do not attempt to quantify specific spectral features using other IR filters here. We have thus used the broadband F435W and F814W filters, as well as the F606W whenever it was possible. For one of the systems in our sample, MS 2137, the data in the F606W band lacked the superb quality of the rest of the observations, and, to maintain a similar quality level for the sample, we decided to use the F625W filter instead. In any case, to test for possible biases due to this choice, for some clusters, the ICL fractions for these two intermediate-wavelength filters have been estimated as a proxy to show the difference in the ICL fraction between them. Although the FF data are deeper than those from CLASH, the excellent quality of the CLASH data, with a $5 \sigma$ limiting $\mathrm{AB}$ magnitude ranging from $27.2(\mathrm{~F} 435 \mathrm{~W})$ to $27.7 \mathrm{mag}(\mathrm{F} 814 \mathrm{~W})$, guarantees that the ICL can be safely measured with these observations and compared to that obtained from the FF images.

For both CLASH and FF, we used the combined drizzled $H S T$ mosaics that have been created for these projects. These mosaics have been produced in a two-step process, where the first step in all cases begins with the individual raw exposures that are processed with the calibration pipeline $\mathrm{CALACS}^{37}$ at STScI, which includes corrections for bias, dark current, flat field, nonlinearity, charge transfer efficiency losses, and electronic gain and photometric calibration. For CLASH, these exposures were then subsequently aligned, corrected for geometric distortion, cosmic-ray rejected, and combined using the MosaicDrizzle pipeline (Koekemoer et al. 2002, 2011) to produce mosaics with a pixel scale of $0 . \prime 065$ pixel $^{-1}$. The FF mosaics were similarly processed, following the same techniques, with the pixel scale being $0{ }^{\prime \prime} 060$ pixel $^{-1}$ and using the Drizzlepac software tools that were developed by Gonzaga et al. (2012). These fully calibrated high-level products can be retrieved from the Mikulski Archive for Space Telescopes. ${ }^{38}$ Despite the fact that the Bullet cluster does not belong to the CLASH or FF samples, the CLASH team reduced and combined HST/ACS observations of this cluster too, putting them at the collaboration's disposal. For the western subcluster of the Bullet system, we did not find observations in the $\mathrm{F} 435 \mathrm{~W}$ filter, and this is the reason we will not provide the ICL fraction measurement in this band. Even though the images in the three filters were available for the eastern subcluster, the contamination from a nearby, very bright star made it impossible to obtain reliable results, so that subcluster was excluded from the analysis.

As we need to measure the total luminosity of the cluster to estimate the ICL fraction, cluster galaxy members must be

\footnotetext{
37 http://www.stsci.edu/hst/acs/performance/calacs_cte/calacs_cte.html

38 https://archive.stsci.edu/prepds/frontier/
}

Table 1

Spectroscopic Redshift Information Available for Each Cluster, Number of Galaxy Members after Applying the Cluster Membership Algorithm, and Bibliographic Sources

\begin{tabular}{lcccl}
\hline \hline Cluster & $z$ & No. of Spectra & No. of Members & Source \\
\hline A383 & 0.187 & 1420 & 254 & 1,2 \\
A611 & 0.288 & 1202 & 158 & $1,3,4$ \\
MS 2137 & 0.313 & 1874 & 408 & 1,2 \\
MACS 1115 & 0.352 & 1681 & 487 & $1,2,4$ \\
RXJ 2129 & 0.234 & 1654 & 184 & 3 \\
A209 & 0.206 & 1037 & 528 & 1,2 \\
A2744 & 0.307 & 1518 & 348 & 1,7 \\
MACS 0416 & 0.396 & 4386 & 643 & 6 \\
MACS 0717 & 0.548 & 1267 & 581 & 1,5 \\
MACS 1149 & 0.544 & 617 & 311 & 1,5 \\
Bullet (eastern) & 0.296 & 112 & 64 & 1 \\
\hline
\end{tabular}

Notes. References: (1) NED, (2) VLT/VIMOS, (3) Hectospec, (4) SDSS/ BOSS, (5) Ebeling et al. (2014), (6) Balestra et al. (2016), and (7) Owers et al. (2011).

identified. Given the deep, high-quality images of our clusters, we require spectroscopic information to identify the cluster members accurately and thus derive precise ICL fractions. We prefer just using spectroscopic redshifts to avoid the larger uncertainty and the interlopers that cluster membership algorithms based on photometric redshifts entail. Thus, the clusters in our sample were chosen for having not only reliable estimates of their dynamical state but also enough spectroscopic information. Table 1 shows the number of reliable spectroscopic redshifts publicly available for each cluster in our sample, as well as the source of these data. For the redshifts provided by the NASA/IPAC Extragalactic Database (NED), ${ }^{39}$ we have rejected those with photometric or poor quality. Table 1 also shows the number of galaxy members confirmed by our two-step cluster membership algorithm (see Section 4 and Jiménez-Teja \& Dupke 2016 for further information on the classification procedure). Initially, our sample also included the CLASH cluster MACS 1931.8-2635, but after applying the cluster membership criteria, we discovered that too few galaxies with spectroscopic redshift lay on the field of view of the CLASH observations; therefore, it was discarded.

In order to know how the use of spectroscopic redshifts affects our results, we will examine the limiting magnitudes of the spectroscopic surveys used in this work. Given that for each cluster, we have different sources contributing to the final spectroscopic sample (see Table 1), with the deepest data completing the previous spectroscopic surveys, we have determined that the worst-case scenario is presented by the cluster MACS 0717 (excluding the Bullet cluster). This cluster not only has the shallowest spectroscopic sample $(r<21.2$ in the rest frame; Ebeling et al. 2014), but it is also the one with the highest redshift $(z \sim 0.548)$. Calculating the distance modulus for this redshift, $\mathrm{DM}=42.50$, we determine that our spectroscopic sample is complete up to an absolute magnitude of $M_{r}=21.2-42.50=-21.3 \mathrm{mag}$ for cluster MACS 0717. Analyzing the luminosity functions calculated by Connor et al. (2017) using photometric redshifts and assuming a completeness and purity of $100 \%$ for the cluster galaxy members so derived, we estimate that our total luminosity might be underestimated by $\sim 19.7 \%$, for our

\footnotetext{
39 https://ned.ipac.caltech.edu/
} 
worst-case cluster. For the best system in our sample, cluster A383 - which has the lowest redshift and spectra from VLT/ VIMOS - a similar analysis yields an underestimation of its total luminosity of $\sim 0.2 \%$. We will analyze in Section 6 the impact of this possible underestimation in the final ICL fractions.

\section{CICLE}

In Jiménez-Teja \& Dupke (2016), a new algorithm called CICLE was developed to study the ICL and estimate the ICL fraction. The motivation was the need to find a reliable and efficient algorithm able to disentangle the ICL from the light in cluster galaxies without assuming any a priori hypothesis. Traditional methods assume certain characteristics of the ICL, such as its surface brightness, density, distance to the brightest galaxies in the cluster, a symmetric morphology, or a certain radial profile. Instead, CICLE uses the CHEFs (Jiménez-Teja \& Benítez 2012) to model the light surface distribution of all the galaxies in the image to obtain ICL and background maps.

The CHEFs are mathematically orthonormal bases optimized to fit the two-dimensional light distribution of the galaxies. They are built in polar coordinates using Chebyshev rational functions to model the radial coordinate and Fourier modes to expand the angular component. The Chebyshev rational functions inherit the excellent properties of the Chebyshev polynomials, which have been proven to provide optimal interpolations for smooth functions (Boyd 2000; Mason \& Handscomb 2003). In addition, these approximations are readily computed, since the Chebyshev basis is very compact, and we just need a few components to fit a profile with high precision (Jiménez-Teja \& Benítez 2012). The Fourier modes, perfect to interpolate periodic functions, make it possible to fit any galaxy morphology without requiring rotational symmetry. Thus, CICLE creates a two-dimensional CHEF model for every galaxy in a cluster image, including the $\mathrm{BCG}$, to later remove it. Stars are just masked out.

Although fitting a regular galaxy is straightforward for the CHEFs, the case of the BCG is more complex, since it is difficult to know where the halo of the BCG ends and the ICL starts. For this reason, the BCG requires a differentiated treatment (Jiménez-Teja \& Dupke 2016). After removing the $\mathrm{CHEF}$ models for all galaxies, CICLE reinserts the CHEF model of the BCG. In this way, this central area is completely restored, and we obtain an image composed just of the BCG, ICL, and background. To estimate the limits of the light belonging to the BCG, a curvature map is calculated for the entire image. The curvature parameter is a characteristic of each pixel, and it represents the change in slope of a surface at a certain point in every direction. CICLE only assumes that the BCG and ICL profiles have different slopes, otherwise it could be impossible to disentangle them. Under this assumption, the bidimensional limits of the BCG are defined by the points where the slope changes most, estimated through the curvature parameter. Once the outline of the BCG is computed, a new model for the BCG is built within the area delimited by its boundary. After removing this model, an image containing just ICL and background is obtained. We refer the reader to Jiménez-Teja \& Dupke (2016) for a more detailed description of CICLE.

In Jiménez-Teja \& Dupke (2016), the background level was estimated using images of nearby fields, obtained at approximately the same epoch under the same observational characteristics. Since this is not possible for all of the clusters in our current sample, and in many cases, there are no blank areas in the images to measure the background, we decided to use a homogeneous (albeit not so precise) approach to be able to draw a consistent comparison between the final ICL fractions. We used the software SExtractor (Bertin \& Arnouts 1996) to estimate the background map for each cluster in our sample, using exactly the same background parameters. First, the background map is iteratively estimated through a $\kappa \sigma$ clipping algorithm in each mesh of a grid that covers the whole image. The size of each mesh is defined by the parameter BACK_SIZE, and it is one of the most influential parameters in the final background estimation. As a general rule, BACK_SIZE must be higher than the average size of the objects in the image, otherwise some flux from these objects could be absorbed in the background. It cannot be too large, either, because smallscale variations of the background would be erased. However, as we are trying to disentangle the background from the diffuse extended light in the intracluster medium, small fluctuations are not as important as avoiding the contamination of light from the cluster galaxies and the ICL, so we set BACK_SIZE $=512$ (Holwerda 2005).

The grid of values yielded by this algorithm was later smoothed by applying a median filter of size BACK_FILTERSIZE. Again, as we were more interested in the average trend of the background than in small features, we chose a large filter of $5 \times 5$ pixels to smooth out any possible contamination from galaxies, stars, and ICL. Then, this filtered grid was fit by applying a bicubic-spline interpolation, which was later refined by recalculating the background locally around the objects in the image. For each object, the background was estimated in an annulus centered on it, with thickness set to BACKPHOTO_SIZE $=24$, which is the typical value for this parameter (Holwerda 2005).

With this approach, we intended to obtain a background map for each cluster, estimated consistently to allow for a fair comparison of the final results. As can be noticed, our selection of values for the background-related parameters was very different from the usual SExtractor configuration, and some of them were intentionally high, with the aim of insuring, to the extent possible, that the background maps were smooth enough and did not contain any light from the galaxies or the ICL.

Finally, in order to measure the ICL fraction once we have a background-free ICL map, we created an image of the cluster removing the $\mathrm{CHEF}$ models of the foreground and background galaxies. As described in Jiménez-Teja \& Benítez (2012), the cluster membership is determined in a two-step process, the PEAK+GAP algorithm (Owers et al. 2011), using the spectroscopic data available for each system. This composite method first identifies the peak of the cluster in the redshift space and selects a redshift window wide enough to contain the whole distribution of velocities assigned to that peak. Implicitly, the size of this window is proportional to the velocity dispersion of the clusters: merging clusters, with a more scattered velocity distribution, will need a wider window, compared to relaxed systems. This crude selection of cluster member candidates is obviously prone to contamination by interlopers. So, we further refine it using the shifting gapper method (Fadda et al. 1996; Girardi et al. 1996; Boschin et al. 2006; Owers et al. 2011), which uses velocity and spatial information on the candidates simultaneously. The shifting gapper method spatially distributes the candidates according to their clustercentric distance in radial bins. The mean velocity of 
the candidates within each bin is calculated, and those candidates with velocities that are too far from the others are rejected. As unrelaxed clusters are more likely to have a broader spatial distribution, this procedure naturally allows candidates at larger distances to be identified as cluster members for these systems. These two steps are, thus, essential to guarantee that our cluster membership algorithm implicitly takes into account the dynamical state of the systems and does not bias the measurement of their total luminosity, while minimizing contamination by interlopers at the same time. We refer the reader to Jiménez-Teja \& Benítez (2012) for further information on the cluster membership selection algorithm.

\section{ICL in CLASH and FF Clusters: Previous Results}

The properties of the ICL in the CLASH and FF clusters have already been extensively studied by several authors using different techniques. We will briefly describe their results for different subsamples of the CLASH (Presotto et al. 2014; Burke et al. 2015; DeMaio et al. 2015, 2018) and FF clusters (Krick \& Bernstein 2007; Montes \& Trujillo 2014, 2018; Morishita et al. 2017). The work by Presotto et al. (2014) was focused on the CLASH cluster MACS J1206.2-0847, $z \sim 0.44$, which does not belong to our sample. They used deep multiband Subaru data to study the ICL properties. Light from cluster galaxies and foreground and background objects was modeled using traditional analytical profiles, such as single or double Sérsic functions, masking out the galaxies with poor fits. To disentangle the BCG from the ICL, they fit a composite de Vaucouleurs plus Sérsic model, yielding a final ICL fraction of $4.3 \% \pm 0.2 \%$ at $R_{500}$ for the Rc band ( $\left.\lambda_{0}=6550 \AA\right)$. They compared this result with that obtained using a surface brightness threshold to identify the ICL, concluding that this method yields very different ICL fractions, depending on the value of this threshold, and systematically higher than that from the fitting technique. Assuming a surface brightness level of $\mu_{R_{c}}=29.87 \mathrm{mag} \mathrm{arcsec}^{-2}$ (equivalent to $\mu_{V}=27.5 \mathrm{mag} \operatorname{arcsec}^{-2^{-}}$at $z=0$ ), they got an ICL fraction of $4.7 \% \pm 0.4 \%$.

DeMaio et al. (2015) studied the ICL in the IR for four CLASH clusters with $0.44 \leqslant z \leqslant 0.57$, with MACS 1149 being the only one that we have in common in our sample. The ICL radial profile is measured from an ICL map obtained by masking out the light from the galaxies either using the SExtractor segmentation maps or by eye. Three of the clusters showed a significant radial gradient toward bluer color at larger cluster radii (MACS 1149 among them), interpreted as a gradient in metallicity assuming a fixed age for the ICL. The color of the ICL in the fourth cluster was found to have a flat distribution. This study was later continued by DeMaio et al. (2018), analyzing a larger sample composed of 20 clusters drawn from the CLASH set plus seven groups from $H S T$ program \#12575, aiming to study the ICL colors and progenitors as a function of the halo mass. They obtained similar radial color gradients to those in DeMaio et al. (2015) and did not find a statistical difference between clusters and groups. They did find a higher $\mathrm{BCG}+\mathrm{ICL}$ mass fraction (assuming a fixed mass-to-light ratio) for groups than for clusters, as well as a more efficient ICL formation mechanism for low-mass halos within a radius of $100 \mathrm{kpc}$. In both papers, they concluded that the ICL formation is primarily driven by tidal stripping of the outskirts of massive galaxies $\left(M_{\star}>10^{10.4} M_{\odot}\right)$. No ICL fractions were reported.
A subsample of 23 clusters from CLASH was analyzed by Burke et al. (2015) using the technique of thresholding the surface brightness of the ICL $\left(\mu_{B}=25 \mathrm{mag} \mathrm{arcsec}^{-2}\right)$ and masking out the stars and nonmember galaxies with circles of radius proportional to their areas. They were able to estimate the ICL fraction in 13 out of the 23 clusters, reporting values between $\sim 2 \%$ and $23 \%$. They found that their ICL fractions strongly correlate with redshift, independently of the dynamical state of the clusters, growing by a factor of $\sim 4-5$ in $0.18 \leqslant z \leqslant 0.90$ versus the $\sim 1.4$ growth factor found for the BCG from the accretion of its companions in the same redshift range. They concluded that the evolution of the ICL is mainly driven by minor mergers at low redshifts $z \sim 1$, as opposed to the BCG, which is primarily evolving at higher redshifts.

Montes \& Trujillo (2014) processed the images of the first FF cluster observed, A2744, using the rest-frame colors $g-r$ and $i-J$. They measured the ICL using three different estimates: the rest-frame surface brightness in the $J$ band of the ICL $\left(\mu_{J}\right)$, the logarithm of the stellar mass density $(\log (\rho))$, and the radial distance to the most massive galaxies of the cluster $(R)$. The corresponding thresholds established for each parameter were $24 \mathrm{mag} \mathrm{arcsec}^{-2}<\mu_{J}<25 \mathrm{mag} \operatorname{arcsec}^{-2}, \log (\rho)<1.2$, and $R>50 \mathrm{kpc}$, yielding ICL fractions of $5.1 \%, 4.0 \%$, and $10.5 \%$, respectively, within a radius of $400 \mathrm{kpc}$. They also used these two colors to study the properties of the stellar populations in the ICL, finding clear negative radial gradients for both age and metallicity toward the outskirts of the cluster. Their results suggest that the ICL in A2744 is mainly formed by the disruption of infalling satellite galaxies with similar mass $\left(M_{\star} \sim 3 \times 10^{10} M_{\odot}\right)$ and metallicity as the Milky Way, being on average $\sim 5$ Gyr younger than the most massive galaxies of the system. This cluster had its ICL fraction previously calculated by Krick \& Bernstein (2007), using ground-based data observed by the du Pont $2.5 \mathrm{~m}$ telescope at Las Campanas Observatory in two filters, the Gunn $r\left(\lambda_{0}=6550 \AA\right)$ and $V$ $\left(\lambda_{0}=5400 \AA\right)$. Krick \& Bernstein (2007) defined the ICL in these two bands using the rest-frame surface brightness thresholds of $\mu_{r}=26.4$ and $\mu_{V}=26.1 \mathrm{mag} \operatorname{arcsec}^{-2}$, respectively, yielding corresponding ICL fractions of $11 \pm 5$ and $14 \pm 5$ within one-quarter of the virial radius. They found the ICL distribution to be multi-peaked, with a color significantly redder than the red cluster sequence.

The work by Montes \& Trujillo (2014) with A2744 was later expanded to the whole FF sample in Montes \& Trujillo (2018). The ICL stellar population properties are defined using distance criteria, assuming that the ICL is the luminous component that extends beyond a radius of $50 \mathrm{kpc}$ once the galaxies in the image are masked using the segmentation maps provided by SExtractor. Under this definition, they found that the metallicity of the ICL for these six clusters is subsolar, on average, and that its mean stellar age is between 2 and 6 Gyr younger than the most massive galaxies in the systems. They confirmed the stripping of $M_{\star}>10^{10} M_{\odot}$ galaxies to be presumably the principal driver of the ICL formation, occurring at $z<1$. To measure the ICL fraction, they followed an approach similar to that developed for the A2744 cluster, setting a surface brightness threshold of $\mu_{V}=26 \mathrm{mag} \operatorname{arcsec}^{-2}$, which yields ICL fractions in the range of $\sim 1 \%-4 \%$ in the $V$ band for all FF clusters. In order to include the effect of the ICL flux that lies (in projection) within the area dominated by the BCG (defined as $r<50 \mathrm{kpc}$ ), Montes \& Trujillo (2018) linearly interpolated their ICL surface brightness profiles. The new ICL fractions ranged between $\sim 4.8 \%$ and $13 \%$ within the $R_{500}$ radius, with a mean of 
$\sim 7 \%$, with their most relaxed cluster presenting marginal evidence of having a higher ICL fraction compared to the other systems in the sample. Contrary to Burke et al. (2015), they did not find any correlation of the ICL fraction with redshift.

The properties of the ICL in the six FF clusters were also studied by Morishita et al. (2017) but with a completely different method. The brightest galaxies in the images $\left(m_{\mathrm{F} 160 \mathrm{~W}}<26\right)$ were fitted using single Sérsic profiles plus a constant sky component in fixed-size "postage stamps" of $300 \times 300$ pixels. The constant sky level within these boxes was identified with the local ICL. A global ICL map was built as the weighted mean of all the overlapping boxes. The BCG did not receive a differentiated treatment, as its light is disentangled from the ICL as any other luminous galaxy in the field. Analyzing the colors of these ICL maps out to $R \leqslant 300 \mathrm{kpc}$, they observed a radial gradient toward blue at larger cluster radii, as in previous studies. They also calculated the stellar mass distribution of the ICL, finding that the ICL is primarily dominated by moderately old stellar populations between $\sim 1$ and $3 \mathrm{Gyr}$ old that could have been stripped from quiescent cluster galaxies with $M_{\star}<10^{9.5} M_{\odot}$ plus an $\sim 5 \%-10 \%$ fraction of younger stars (A- and earlier-type, $\sim 1 \mathrm{Gyr}$ ) at $R \leqslant 150 \mathrm{kpc}$ presumably coming from recently star-forming/ infalling galaxies. Although ICL light fractions are not computed, they reported ICL mass fractions calculated from SED fitting for all six clusters, ranging between $\sim 7 \%$ and $23 \%$ within a radius of $300 \mathrm{kpc}$ and $\sim 4 \%-19 \%$ for $R \leqslant 500 \mathrm{kpc}$. Again, no trend can be identified with redshift, as in Montes \& Trujillo (2018).

ICL or ICL+background maps of CLASH and FF clusters have also been obtained as by-products in other works with photometric or gravitational-lensing purposes, although neither the ICL properties nor the ICL fraction are studied in these works (e.g., Merlin et al. 2016; Connor et al. 2017; Livermore et al. 2017; Molino et al. 2017).

\section{Results with CICLE}

We estimated the ICL fraction of our sample of 11 massive clusters in three ACS/WFC broadbands (F435W, F606W, and F814W) whenever possible. In the case of MS 2137, the F606W observation from CLASH was not as good as the rest of the data, so we decided to process the F625W filter instead. We also estimated the ICL fraction in the F625W filter (as well as in the F606W band) for one of the merging clusters (MACS 0416) and one of the relaxed systems (A383), just for comparison. In the case of the Bullet cluster, we only estimated the ICL fractions for the eastern subcluster, since the measurements for the western subcluster were polluted by the presence of a nearby very bright star. Unfortunately, we did not have data for the eastern subcluster in the F435W filters, and that is why we report the ICL fractions just in the F606W and F814W bands.

The images were first preprocessed to mask out the brightest stars, since they are not smooth enough to be processed by the CHEFs (Jiménez-Teja \& Dupke 2016). We masked the areas associated with these stars in the SExtractor segmentation map for the F814W band, enhanced using a $10 \times 10$ pixel filter. The same mask is applied to the other two filters, F435W and F606W, to ensure that the differences in the ICL fractions are physical and not induced by different masking. These masked pixels are excluded from the final measurement of the ICL fraction.

Then, we run CICLE to obtain ICL+background maps (see Section 4). The original images and the resulting maps for each cluster in the different filters are displayed in Appendix A: Figures 3-7 for the relaxed subsample and Figures $8-13$ for the unrelaxed systems. The typical value of the background found for our whole sample ranges from approximately (7-8)e-05 cps for the three main filters considered, completely consistent with the values calculated by Morishita et al. (2017) using a different algorithm. These background values represent $\sim 33 \%(\mathrm{~F} 435 \mathrm{~W})$, $\sim 9.0 \%(\mathrm{~F} 606 \mathrm{~W})$, and $\sim 8.5 \%(\mathrm{~F} 814 \mathrm{~W})$ of the ICL flux. Using the $r_{200}$ radii reported by Boschin et al. (2006), Maier et al. (2016), Martinet et al. (2017), and Morishita et al. (2017), we calculated the apparent size of the clusters in our sample. We found no correlation between the background values measured in the three main filters and the apparent size of the clusters, guaranteeing that our background measurements are not biased.

We then computed the radial flux profiles of the ICL surface. As in Jiménez-Teja \& Dupke (2016), these radial profiles are obtained by averaging the flux inside the natural contours of the ICL in the core of the cluster and inside ellipses in the outskirts. These radial profiles show a negative slope reaching a minimum from which the ICL flux starts to increase due to the instrumental light from the borders of the images, or where the ICL submerges into the background. We thus measured the ICL fraction up to that radius, where the flux profile is minimum, and beyond which our estimation would be unreliable due to spurious instrumental effects, as described in Jiménez-Teja \& Dupke (2016). Given the similar depths in the filters F606W and F814W for the CLASH data ( $\sim 27.6$ and $\sim 27.7 \mathrm{AB}$ mag for a $5 \sigma$ point source within a 0.4 diameter aperture, respectively; Postman et al. 2012), we can presume that difference in the ICL radii between these two filters is physical. However, the F435W depth is $27.2 \mathrm{AB}$ mag, which is why observational characteristics might be the cause of the different areas. As for the FF images, the F435W and F606W depths are virtually the same, $\sim 28.8 \mathrm{AB}$ mag, while $\mathrm{F} 814 \mathrm{~W}$ is slightly deeper ( 29.1 AB mag; Merlin et al. 2016; Lotz et al. 2017).

We summarize in Table 2 the resulting ICL fractions and the corresponding radii of the measurements. The errors associated with the ICL fractions were estimated as the quadratic sum of the photometric error of the measured flux and the intrinsic error of the CICLE algorithm in the disentanglement of the BCG from the ICL. The former error is negligible in comparison to the latter in most cases, due to the high signal-to-noise of the HST images. However, as we described in Section 3, the use of spectroscopic redshifts for the cluster membership could cause a possible underestimation of $\sim 19.7 \%$ in the total luminosity of the cluster, for the worst system in our sample (excluding the Bullet cluster). Propagating the errors and using the highest ICL fraction found for this cluster $(22.27 \%$, which yields the largest error associated with this problem), this would imply, if anything, a maximum error of $\sim 4.39 \%$ to be added to the values listed in Table 2 . This upper limit in the error induced by the limiting magnitude of the spectra confirms the advantage of the use of these data, whenever available, instead of photometric information for the cluster membership, since it is small compared with the potential contamination introduced by photometric redshifts in this identification. For the sake of comparison, the underestimation of the total luminosity for our best-case cluster, A383, is approximately $\sim 0.2 \%$, which is translated into an additional ICL fraction error of $\sim 0.02 \%$, which is completely negligible.

As was described in Jiménez-Teja \& Dupke (2016), the second source of error, the error of the CICLE algorithm, was estimated using mock images with the same characteristics as the real data: for each image, we created a simulated image of the same size and containing two exponential profiles with effective radii and surface brightness equal to those of the real BCG and ICL 


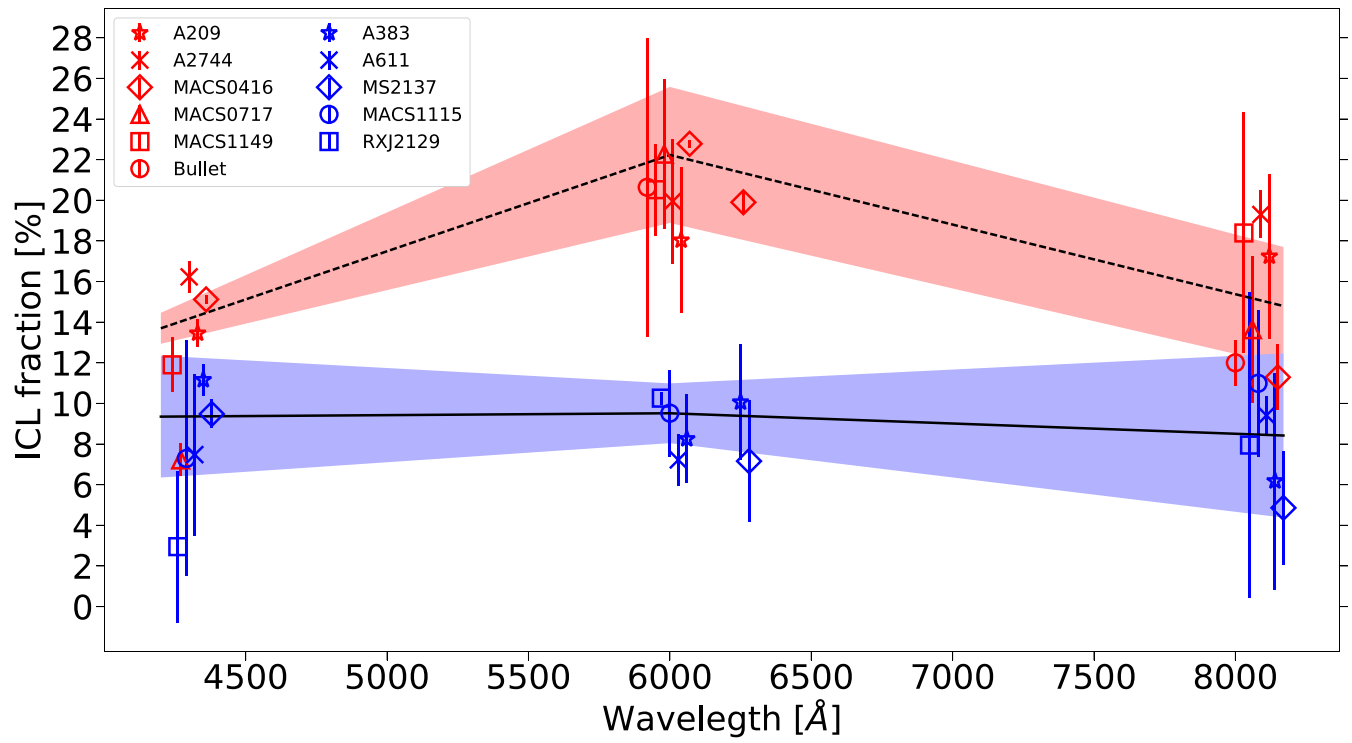

Figure 1. ICL fractions yielded by CICLE for our sample of 11 clusters. Red symbols represent merging clusters, while blue symbols are associated with relaxed systems. The black lines indicate the error-weighted mean for each subsample (solid for relaxed clusters and dashed for merging systems), and the shaded areas represent the mean of the errors. For clarity, we have horizontally offset the points by $30 \AA$ gaps.

Table 2

Results Yielded by CICLE for the 11 Clusters in Our Sample: ICL Fractions and Errors for the Different Filters and the Radii Used to Measure These ICL Fractions

\begin{tabular}{|c|c|c|c|c|c|c|c|c|}
\hline \multirow[b]{2}{*}{ Cluster } & \multicolumn{2}{|c|}{$\mathrm{F} 435 \mathrm{~W}$} & \multicolumn{2}{|c|}{ F606W } & \multicolumn{2}{|c|}{ F625W } & \multicolumn{2}{|c|}{ F814W } \\
\hline & $\begin{array}{c}\text { ICL Fraction } \\
(\%)\end{array}$ & $\begin{array}{c}\text { Radius } \\
(\mathrm{kpc})\end{array}$ & $\begin{array}{c}\text { ICL Fraction } \\
(\%)\end{array}$ & $\begin{array}{l}\text { Radius } \\
(\mathrm{kpc})\end{array}$ & $\begin{array}{c}\text { ICL Fraction } \\
(\%)\end{array}$ & $\begin{array}{c}\text { Radius } \\
(\mathrm{kpc})\end{array}$ & $\begin{array}{c}\text { ICL Fraction } \\
(\%)\end{array}$ & $\begin{array}{c}\text { Radius } \\
(\mathrm{kpc})\end{array}$ \\
\hline A611* & $7.48 \pm 3.98$ & 141.1 & $7.22 \pm 1.26$ & 159.1 & $\ldots$ & $\ldots$ & $9.41 \pm 0.95$ & 252.5 \\
\hline MS $2137^{*}$ & $9.48 \pm 0.71$ & 135.4 & $\ldots$ & $\ldots$ & $7.16 \pm 2.99$ & 179.6 & $4.86 \pm 2.80$ & 316.0 \\
\hline MACS $1115^{*}$ & $7.29 \pm 5.79$ & 163.8 & $9.52 \pm 2.13$ & 252.0 & $\ldots$ & $\ldots$ & $10.99 \pm 3.79$ & 250.5 \\
\hline RXJ $2129^{*}$ & $2.95 \pm 3.74$ & 63.9 & $10.26 \pm 0.31$ & 227.6 & $\cdots$ & $\cdots$ & $7.95 \pm 7.53$ & 176.2 \\
\hline MACS 0416 & $15.12 \pm 0.22$ & 336.8 & $22.78 \pm 0.19$ & 328.3 & $19.90 \pm 0.51$ & 310.6 & $11.29 \pm 1.60$ & 332.8 \\
\hline MACS 0717 & $7.22 \pm 0.81$ & 275.3 & $22.27 \pm 3.68$ & 562.5 & $\ldots$ & $\ldots$ & $13.63 \pm 3.60$ & 421.6 \\
\hline MACS 1149 & $11.90 \pm 1.34$ & 172.5 & $20.52 \pm 2.24$ & 336.2 & $\cdots$ & $\cdots$ & $18.39 \pm 5.91$ & 626.3 \\
\hline Bullet (eastern) & $\ldots$ & $\ldots$ & $20.64 \pm 7.35$ & 217.9 & $\ldots$ & $\cdots$ & $12.00 \pm 1.11$ & 349.5 \\
\hline
\end{tabular}

Note. Systems belonging to the relaxed sample are marked with an asterisk.

surfaces. We then polluted the mock images with 10 realizations of noise with the signal-to-noise ratio of the original observations and applied CICLE to them. The final error was obtained as the mean of the errors of the 10 realizations.

The obtained ICL fractions are plotted in Figure 1, where the merging clusters are represented with red symbols and the regular systems with blue symbols. For clarity, measurements for some clusters have been slightly offset horizontally by $30 \AA$ gaps. We observe that the relaxed systems seem to have a nearly constant gradient in the ICL fraction within the error bars, while the disturbed clusters surprisingly show a clear increase in the F606W ICL fraction. Although, on average, the ICL fractions of the merging sample are higher than those of the regular clusters, we conclude from Figure 1 that the bluest and reddest filters, F435W and F814W, cannot be used to discriminate between relaxed and nonrelaxed systems. However, the ICL fraction in the F606W broadband quantitatively describes a significant difference between the dynamical states of the clusters.

Due to the disparate techniques applied, the different reference filters used, and the different apertures to estimate the ICL fraction, it is difficult to have a direct quantitative comparison of the ICL fractions estimated by us to those in the literature (see Rudick et al. 2011 for a comparison of methods). Our ICL fractions are, in general, comparable to or higher than those reported previously. This is partially explained by the fact that CICLE includes in the estimations the ICL projected over the BCG-dominated area, and this is not the case for surface brightness- and radial distance-based methods. By design, in these traditional techniques, the ICL projected over the central regions is not added up to the final estimation of ICL flux, since these pixels are excluded. This lost flux is of great importance, since the ICL is known to be more concentrated in the central area, and this may cause a significant underestimation of the ICL fraction (Willman et al. 2004; Rudick et al. 2006). Moreover, our ICL fractions are not measured homogeneously up to the same metric radius, but we restrict our calculations to the total area of the aperture that is not contaminated by spurious instrumental light. In many cases, our radii are smaller than those used in the literature, thus yielding higher ICL fractions (Rudick et al. 2006). 


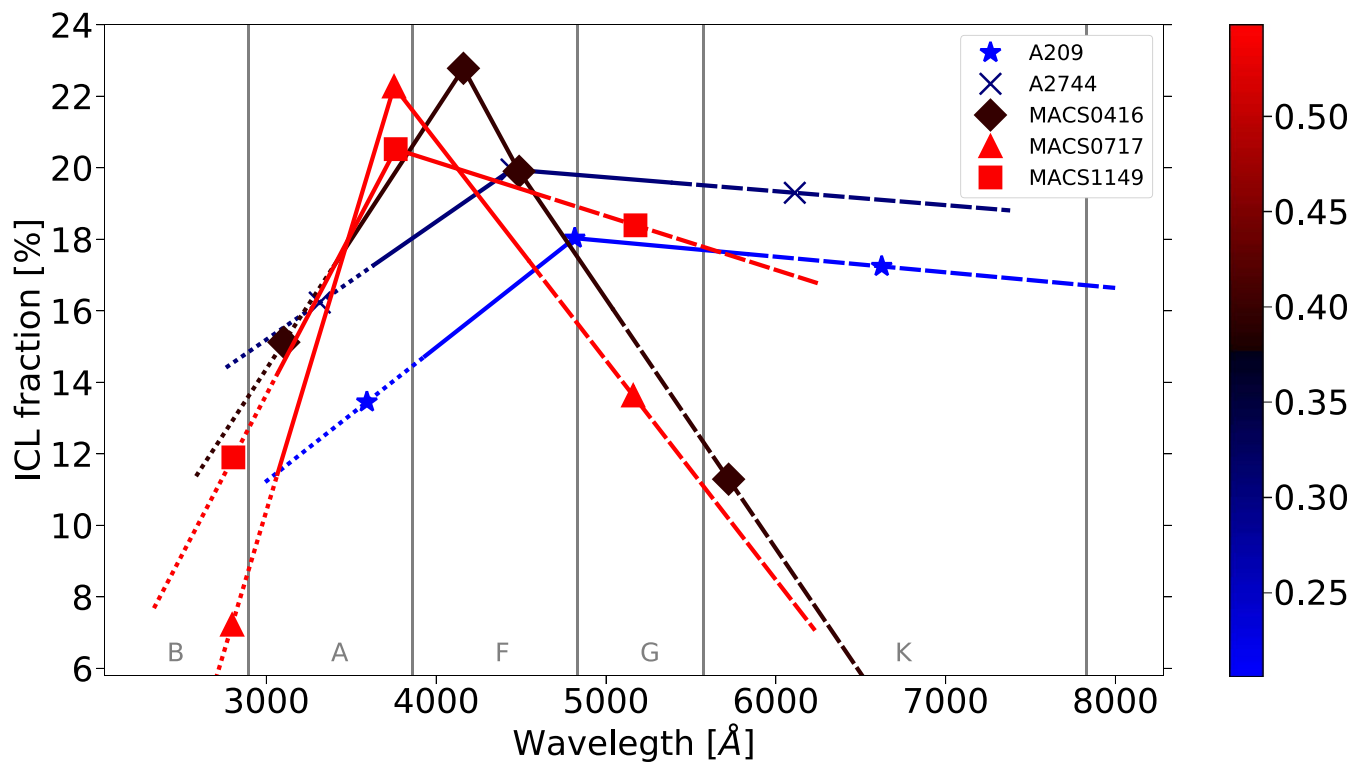

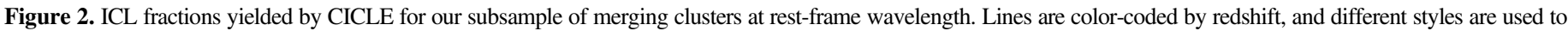

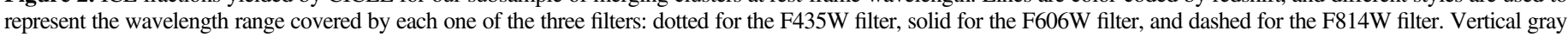
lines separate the wavelength intervals where the emission peaks of the different stellar spectral types are included, as indicated at the bottom of each region.

We do not observe any trend in the ICL fraction with redshift, in contrast with Burke et al. (2015) but in line with the works by Krick \& Bernstein (2007), Montes \& Trujillo (2018), and Morishita et al. (2017). One should notice that, as we are working with ICL fractions and not ICL fluxes, the results shown are robust without the need for applying any correction for redshift or evolution.

\section{Discussion}

A first attempt to link the dynamical state of the clusters with the properties of the ICL was made by Feldmeier et al. (2002), although without quantifying the amount of ICL or the ICL fraction. Similarly to us, Krick \& Bernstein (2007) obtained lower ICL fluxes for apparently more relaxed systems when they analyzed a sample of 10 clusters in the redshift range $0.05 \leqslant z \leqslant 0.3$. Using the M3-M1 parameter (magnitude difference between the first- (M1) and third- (M3) brightest galaxy members of the cluster) as an estimator of the dynamical age of the system, they found that it correlates with the ICL luminosities measured in the broadband Gunn $r$ band (with a central wavelength $\lambda_{0}=6550 \AA$, which can be considered comparable to our ACS F606W filter). They also observed similar relations for other dynamical indicators, as, for instance, the M2-M1 magnitude difference (defined in a similar way as the M3-M1) or simply the presence of single, large elliptical galaxies (cD) in the cluster core. Moreover, the ICL fluxes found for clusters without $\mathrm{cD}$ galaxies are twice as high as those measured in systems with cD galaxies, on average. Interestingly, in spite of using a different sample, data with different observational characteristics, and a different technique, yielding lower ICL fractions than ours, Krick \& Bernstein (2007) observed the same trend. However, as the timescales governing the dynamics of the stars in the ICL are not consistent with settling down into the center of the gravitational potential (or BCG) due to angular momentum or energy losses (Merritt 1984), Krick \& Bernstein (2007) concluded that either stars in the ICL have been formed early in groups that migrate to the center, or they have been directly stripped at the center of the cluster potential at later times, or even that the ICL is observationally indistinguishable from the BCG halo. They also find steeper ICL profiles for relaxed clusters, which would favor the hypothesis of an ICL evolution linked to the BCG formation: as groups are being merged with the BCG, they could bring their primordial ICL stars with them and, at the same time, create even more ICL by ram pressure of the gas or dynamical friction. However, in the absence of a central dominant galaxy, groups and cluster galaxies would evolve slowly by tidal forces and dynamical friction, barely influencing the stars in the ICL and displaying shallower ICL profiles.

Moreover, whereas the energy and angular momentum of the groups dissipate and they bring their ICL stars with them, any ICL formed by galaxy interactions would stay in the orbit where it was formed (Krick \& Bernstein 2007). If this mechanism for producing ICL can be efficient at larger radii, this would explain the ICL radial color gradient reported by several authors (e.g., Montes \& Trujillo 2014; DeMaio et al. 2018), in particular for the systems in the CLASH and FF samples. DeMaio et al. (2018) found that 75\% of the ICL luminosity in the CLASH clusters was consistent in color with the stars stripped from the outskirts of cluster galaxies with $M_{\star}>10^{10.4} M_{\odot}$, while Morishita et al. (2017) observed that 90\%-95\% of the ICL mass in the FF systems had colors that were compatible with the outer regions of quiescent cluster galaxies of $M_{\star}<10^{9.5} M_{\odot}$. Then, if the lower-metallicity/younger stars stripped from the outskirts of the luminous galaxies stay in their orbits, they will create the bluer trend observed toward larger cluster radii. This gradient would also be expected from the contribution to the ICL from the disruption of low-mass, lowmetallicity dwarf galaxies, which are completely shredded at larger cluster radii compared to more massive, metal-rich galaxies (DeMaio et al. 2015, 2018).

Numerical simulations predict a growth in the ICL fraction with decreasing redshift (Willman et al. 2004; Rudick et al. 2006, 2011). However, some authors found that the ICL fraction changes slightly over short timescales (as major mergers or collisions occur). For instance, Willman et al. (2004) predicted that the amount of ICL is directly linked to the infall of large groups already containing unbounded ICL stars, although they also find that this does not necessarily change the ICL fraction. Rudick et al. 
(2006) made a very detailed study of the effect of the dynamics on the ICL fraction in three different clusters, concluding that the ICL fraction growth is mainly driven by accretion events of massive galaxies and groups falling into the cluster center. For those systems that did not experience major interactions, the ICL fraction evolved passively, rising slowly. However, it is interesting that, although the amount of ICL increases dramatically in mergers (potentially even doubling the luminosity), they observed a decline in the ICL fraction at the beginning of the interaction. This is explained by their definition of ICL through a surface brightness threshold of $\mu_{V}=26.5 \mathrm{mag} \operatorname{arcsec}^{-2}$, which biases their measurements of the ICL fraction in the time preceding a major merger. As the galaxy groups start to infall, the luminosity appears more concentrated and thus temporarily increases the surface brightness of the cluster. Stars belonging to the ICL are therefore boosted and misclassified as part of the cluster galaxies during a short time. At later merging epochs, they did observe a rise in the ICL fraction associated with the merger event, in general agreement with our results. This conclusion was later corroborated by other authors with different numerical simulations (e.g., Murante et al. 2007; Contini et al. 2014).

In general, Rudick et al. (2011) proved that the different definitions of ICL fraction show consistent behaviors, in spite of yielding very different values for the ICL fraction. In particular, the ICL fraction was found to rise irregularly during the cluster evolution due to merging events, which can easily cause the ICL fraction in individual clusters to deviate from the global average trend with redshift. This implies that the ICL fraction alone in a single filter cannot be a robust estimator of the dynamical stage of the clusters, since a merging cluster at higher redshift could have a similar ICL fraction as a relaxed system at lower redshift. However, our findings raise the possibility of using the ICL fraction color instead of the ICL fraction to estimate the dynamical stage of clusters, at least in the case of massive systems.

For our subsample of relaxed systems, we observe that the distribution of the ICL fractions along the different wavelengths is nearly constant within the error bars, except for the case of the cluster RXJ 2129 (see Figure 1). That means that the colors of the ICL are coincident, on average, with those of the stellar populations in the galaxies, considering the cluster as a whole. This is consistent with the idea that these systems have reached a virialized stage, the ICL stellar populations are just evolving passively, and the ICL fraction is slowly fed by the stars stripped out from the cluster member galaxies by dynamical friction. However, for the subsample of merging clusters, the ICL fraction exhibits a strong increase in the $\mathrm{F} 606 \mathrm{~W}$ band, significant at $2.5 \sigma$ with respect to the subsample of relaxed systems. Compared to the increase observed in the other two filters, this excess is especially significant with respect to the F435W filter, given the smaller error bars. So, for clusters suffering major merger events, we see an excess of flux for the ICL in the F606W band compared to the cluster galaxy light, meaning that a significant fraction of bluer stars, presumably with lower metallicities, is being stripped out violently from the outskirts of the infall galaxies. The presence of these stars (Goddard et al. 2016a, 2017b) would cause the ICL to be bluer than the overall light from galaxies, in comparison to relaxed clusters. An interesting question is why this blueing-merger effect is more pronounced systematically in the F606W filter than in the F435 band. In their detailed analysis of the properties of the ICL in the six FF clusters, Morishita et al. (2017) found that the ICL was mainly composed (in mass) of moderately old stellar populations ( 1-3 Gyr), which would contribute more to the F606W filter than to the F435W. However, they also observed a nonnegligible fraction of the ICL stellar mass that was likely associated with a bluer/younger population ( $\sim 1 \mathrm{Gyr})$. They estimated that approximately $5 \%-10 \%$ of the ICL mass was compound of A- or earliertype stars, probably stripped from star-forming galaxies during the cluster merging process. The A-type stars have a lifetime of $\sim 1$ Gyr, on average, which, compared to an average crossing time ( $\sim 1 \mathrm{Gyr}$ ), would make it possible to see the influence of these stars on the ICL fraction. In Figure 2, we can visualize the filters where A-type star flux would contribute the most, according to the redshift of each cluster. The ICL fractions are now plotted at the rest-frame wavelengths, color-coded by the redshift of the clusters, and the line style indicates the wavelength range covered by each filter: dotted lines for the F435W band, solid lines for the F606W filter, and dashed lines for the F814W band. Given that A-type stars display temperatures from 7500 to $10000 \mathrm{~K}$, their peak emission will range from $\sim 2900$ to $3900 \AA$. For the two highest-redshift clusters in our sample, MACS 1149 and MACS 0717, at $z \sim 0.544$ and 0.548, respectively, this emission would be almost completely included in the F606W filter, with little contribution to the F435W flux. For the merging system MACS 0416 at $z \sim 0.396$, the young population flux contribution would be divided between the two filters. However, for the lowest-redshift FF cluster in our sample, A2744 $(z \sim 0.307$ ), A-type stars would be mostly observed in the F435W band, which could presumably explain why the gradient between the F606W and F435W filters in this cluster is not as pronounced as for the rest of the merging subsample. Although the cluster A209 does not belong to the FF sample, and we do not have information on the possible ICL stellar populations, its low redshift $(z \sim 0.206)$ and ICL fraction colors coincident with those of A2744 suggest a similar explanation based on the presence of younger stars.

We must also notice that, in spite of having ICL fractions consistent with those of our relaxed sample, the regular cluster RXJ 2129 displays an ICL fraction color distribution behavior similar to that of the merging sample, with a peak in the F606W. Even though the errors estimated for the F435W and F606W measurements are high, we can presume that the ongoing minor mergers pointed out by other authors (Kale et al. 2015; PandeyPommier et al. 2016; Giacintucci et al. 2017) could be the origin of this fluctuation. This would be an interesting prediction for the relation between dynamical activity in clusters and the color (spectral energy distribution) gradient described in this work. If this is confirmed, the color distribution of an individual cluster's ICL fraction could be used to estimate the mass ratio of mergers solely using optical data.

The relation between ICL fraction color gradient and cluster dynamics described in this work can be extremely useful, not just to select clusters for further analysis of the merging process but also to exclude merging clusters from scaling relations for mass proxies used in cosmology with purely optical data, such as those incoming from the current and near-future megasurveys, such as DES $^{40}$ and J-PAS. ${ }^{41}$

\section{Conclusions}

We have analyzed the ICL in 11 systems with high-quality imaging and enough spectroscopic information available, with the aim of characterizing their dynamical state through the ICL fraction. We have applied CICLE, a new algorithm described in Jiménez-Teja \& Dupke (2016) that is free of a priori

\footnotetext{
40 http://www.darkenergysurvey.org

41 http://www.j-pas.org
} 
assumptions on the properties of both ICL and galaxies. In that work, CICLE was found to estimate the ICL fraction with a maximum error of $10 \%$ in the absence of noise for reasonable configurations of the ICL + BCG system. CICLE was thus proved to be robust and accurate, ideal for consistently studying our sample of $11 H S T$-observed clusters.

The dynamical stage of the clusters was carefully determined by compiling the different results available in the literature and gathering probes on X-ray morphology, dynamical analysis, and radio information. Five of the systems had strong indications of being relaxed systems, while the other six showed clear signs of dynamical activity. The resulting ICL fractions in the three optical broadband filters F435W, F606W, and F814W for the subsample of regular clusters were nearly constant within the error bars, ranging between $\sim 2 \%$ and $11 \%$. For the six merging clusters, we report higher ICL fractions, on average, in the three filters in the interval $\sim 7 \%-23 \%$. A different behavior is displayed in observing the ICL fraction colors, with a significant peak in the ICL fraction measured at the intermediate band. Both the higher ICL fractions and the peak at the F606W band are consistent with previous results in the literature, although derived from simulations or analyses of the ICL colors. No obvious trend is identified in the ICL fraction with redshift.

Although a larger sample of galaxy clusters with clearly defined dynamical states and HST-like observational characteristics is necessary to improve our statistical significance, we have shown that the ICL fraction colors, measured robustly and consistently, can offer valuable information on the dynamical processes occurring in clusters. Since bona fide relaxed systems are more difficult to classify as such than merging systems, the addition of a truly relaxed system ICL fraction measurement would be extremely desirable to establish the range variation of this color gradient with respect to merger stage. In that case, systems that are very old and relaxed, such as fossil groups of galaxies with deep enough observations at comparable redshifts, would be the best candidates, which are currently unavailable in the HST archive.

We thank the referee for constructive comments that helped to improve the original manuscript. Y.J.-T. would like to thank Dr. Marc Postman for his support, help, and encouragement during her stay at the STScI, which made this work possible. We gratefully acknowledge the computational support of Dr. Fernando Roig. Y.J.-T. also acknowledges financial support from the Fundação Carlos Chagas Filho de Amparo à Pesquisa do Estado do Rio de Janeiro (FAPERJ; fellowship Nota 10, PDR-10) through grant E-26/202.835/2016 and the Coordenação de Aperfeiçoamento de Pessoal de Nível Superior (CAPES; Science without Borders program, Young Talent Fellowship, BJT) through grant A062/2013. R.A.D. acknowledges support from the Conselho Nacional de Desenvolvimento Científico e Tecnológico (CNPq) through BP grant 312307/2015-2 and the Financiadora de Estudos e Projetos (FINEP) grant REF. 1217/ 13-01.13.0279.00. Both Y.J.-T. and R.A.D. also acknowledge support from the Spanish National Research Council (CSIC; I-COOP +2016 program) through grant COOPB20263 and the Spanish Ministry of Economy, Industry, and Competitiveness (MINECO) through grants AYA2013-48623-C2-1-P and AYA2016-81065-C2-1-P. K.U. acknowledges support from the Ministry of Science and Technology of Taiwan (grant MOST 103-2628-M-001-003-MY3) and from the Academia Sinica Investigator Award. M.M. acknowledges support from the Italian Ministry of Foreign Affairs and International Cooperation, Directorate General for Country Promotion.

\section{Appendix A ICL+background Maps}

Here Figures 3-7 show the relaxed subsample and Figures 8-13 show the unrelaxed systems.

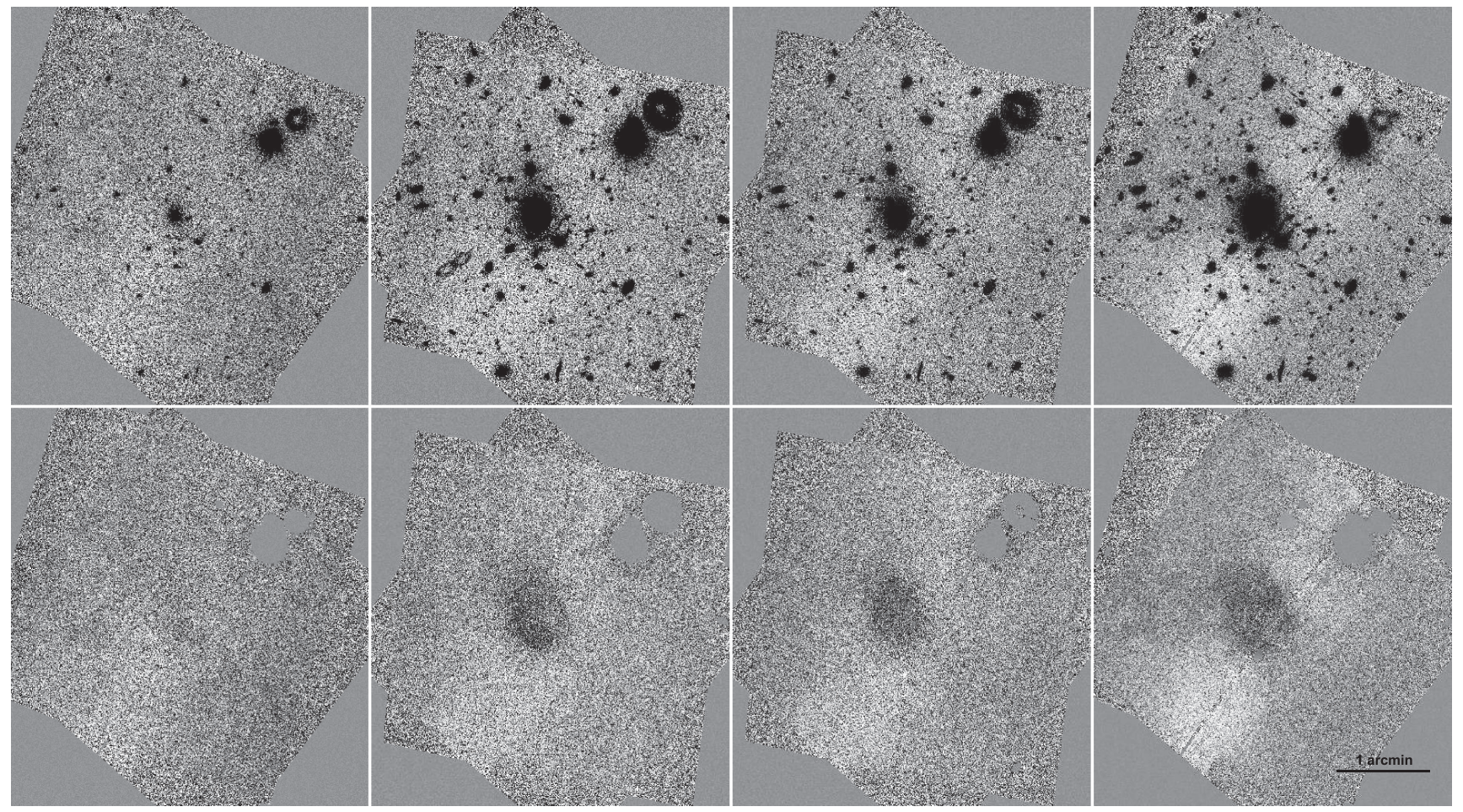

Figure 3. Original images of the relaxed cluster A383 (top) and ICL+background maps provided by CICLE (bottom) in the F435W, F606W, F625W, and F814W filters (from left to right). The scale of the original and ICL+background images is the same for each filter. 

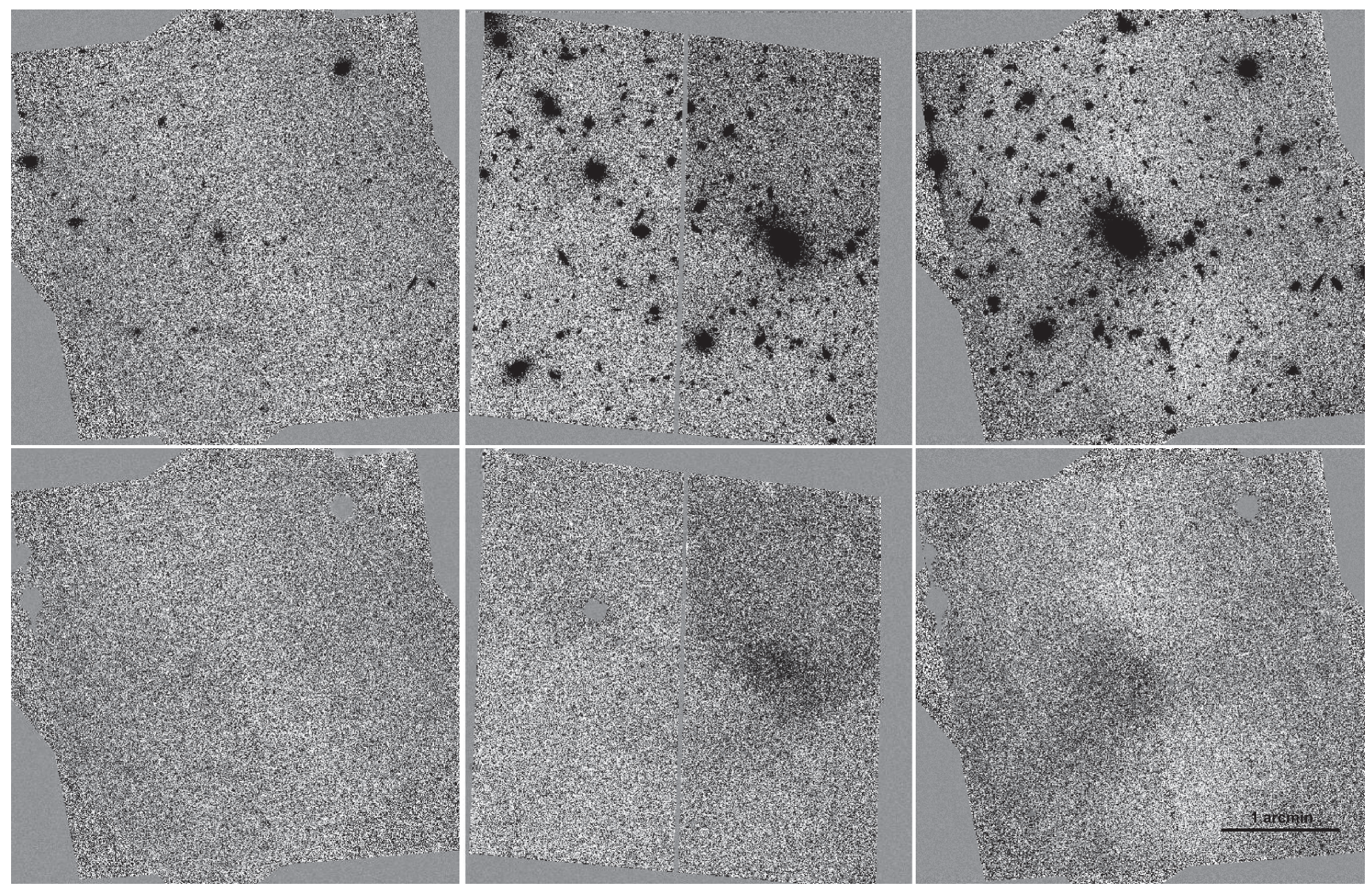

Figure 4. Original images of the relaxed cluster A611 (top) and ICL+background maps provided by CICLE (bottom) in the F435W, F606W, and F814W filters (from left to right). The scale of the original and ICL+background images is the same for each filter.

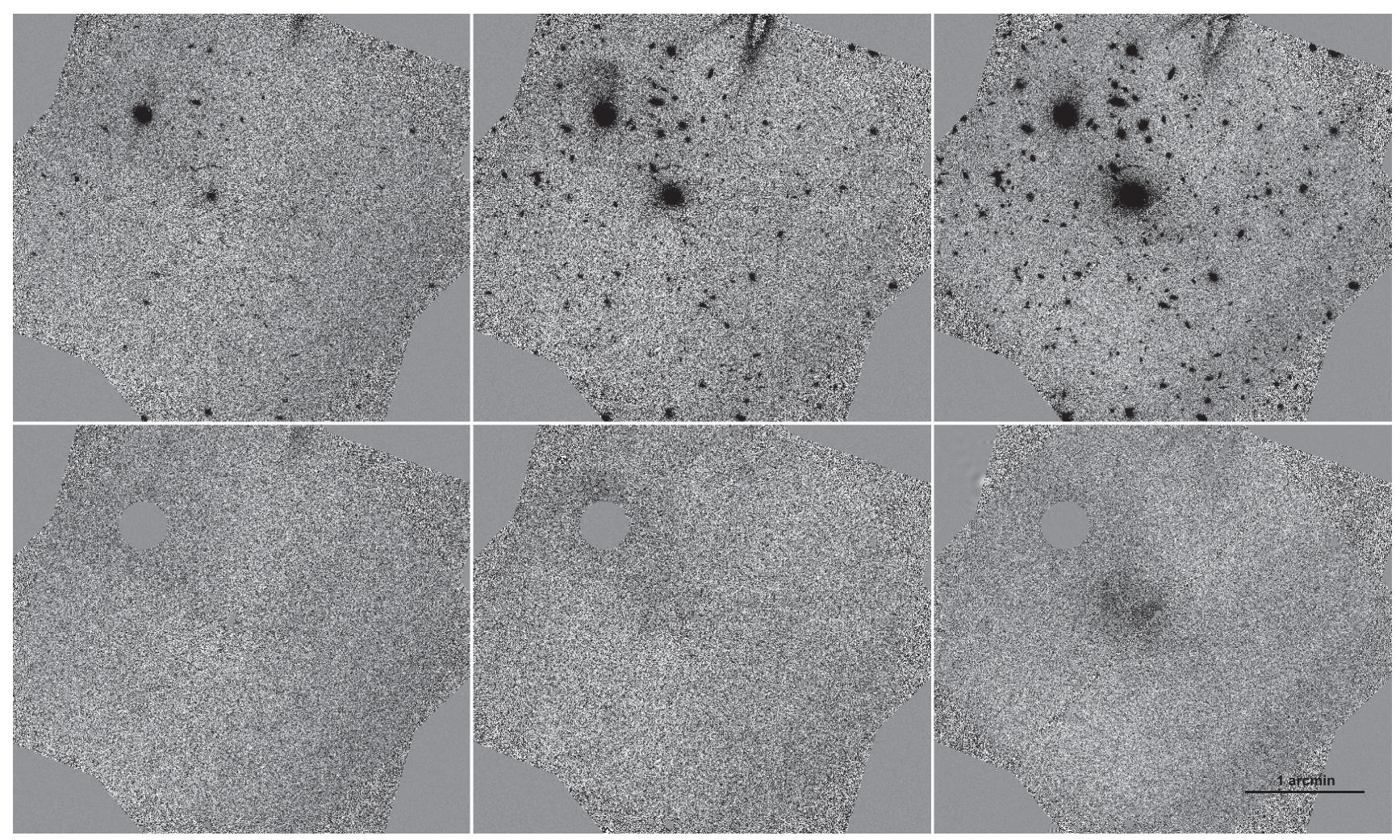

Figure 5. Original images of the relaxed cluster MS 2137 (top) and ICL+background maps provided by CICLE (bottom) in the F435W, F625W, and F814W filters (from left to right). The scale of the original and ICL+background images is the same for each filter. 


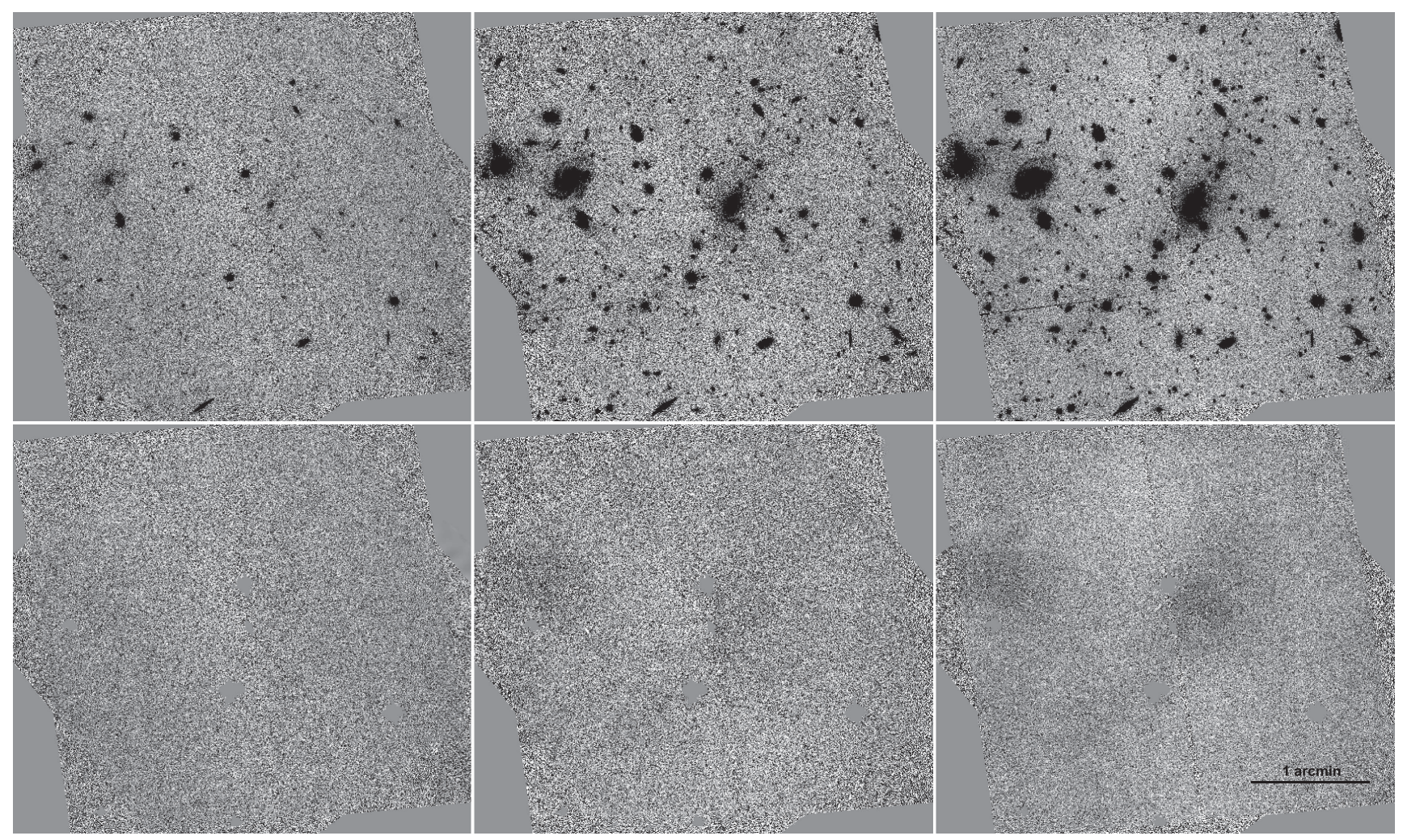

Figure 6. Original images of the relaxed cluster MACS 1115 (top) and ICL+background maps provided by CICLE (bottom) in the F435W, F606W, and F814W filters (from left to right). The scale of the original and ICL+background images is the same for each filter.

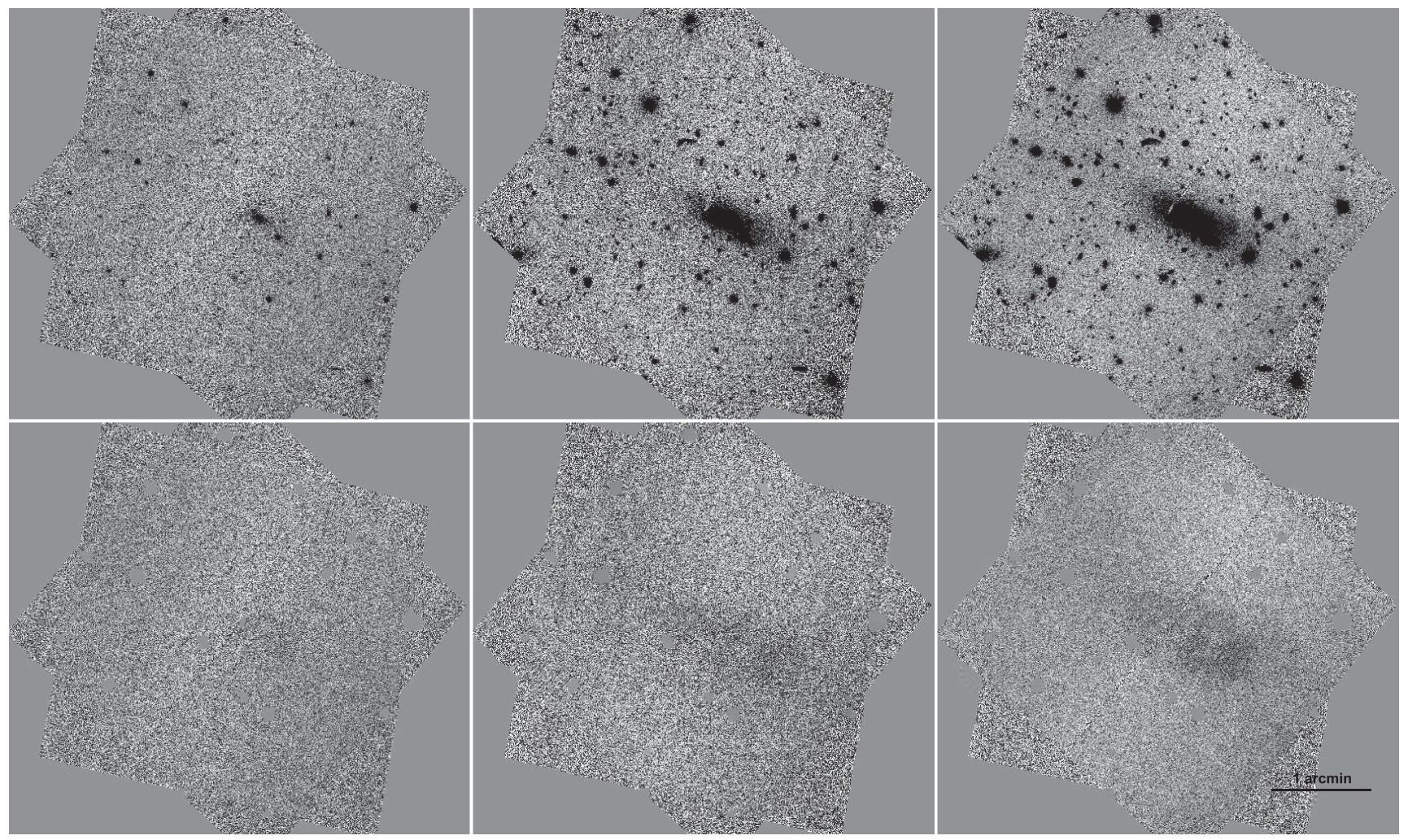

Figure 7. Original images of the relaxed cluster RXJ 2129 (top) and ICL+background maps provided by CICLE (bottom) in the F435W, F606W, and F814W filters (from left to right). The scale of the original and ICL+background images is the same for each filter. 


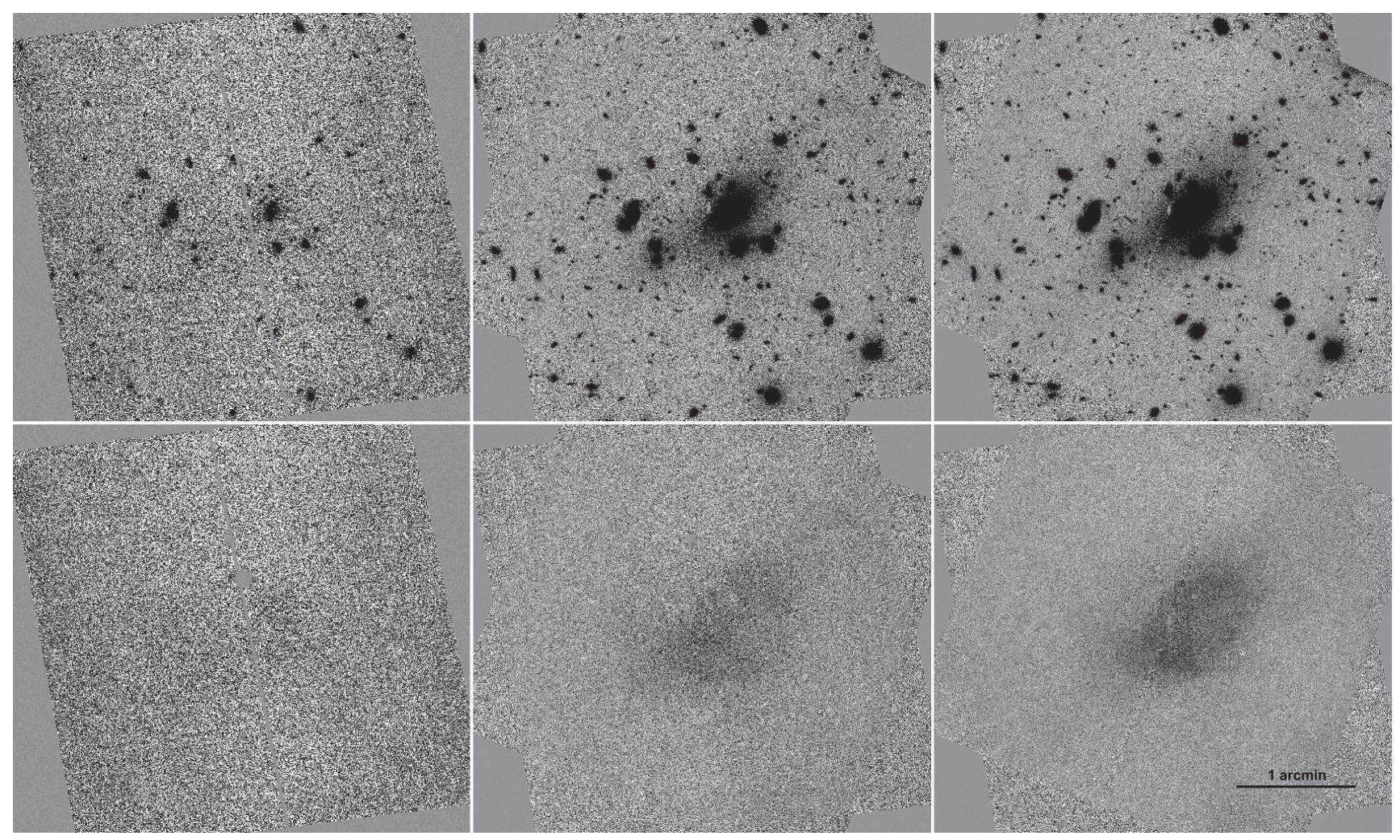

Figure 8. Original images of the unrelaxed cluster A209 (top) and ICL+background maps provided by CICLE (bottom) in the F435W, F606W, and F814W filters (from left to right). The scale of the original and ICL+background images is the same for each filter.

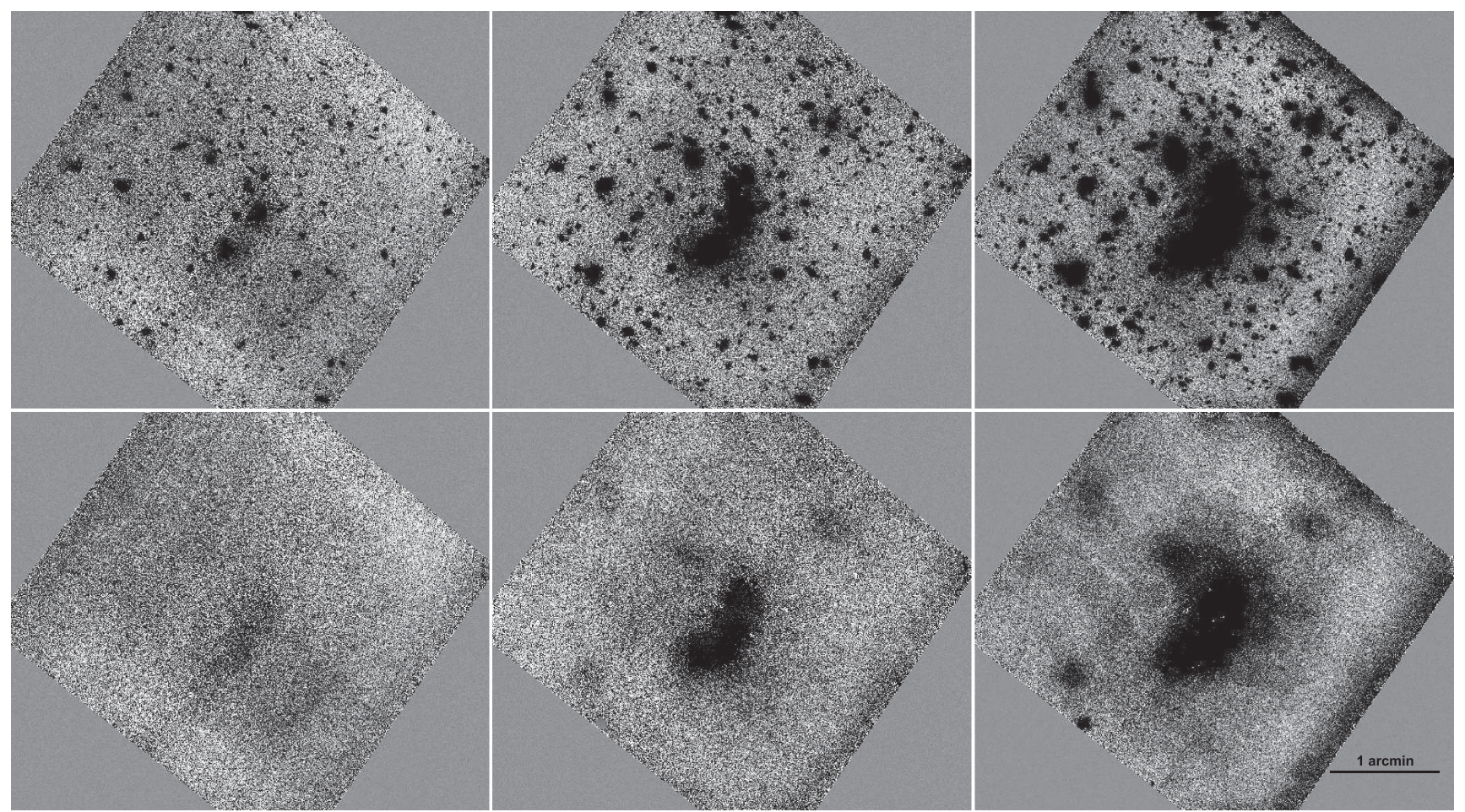

Figure 9. Original images of the unrelaxed cluster A2744 (top) and ICL+background maps provided by CICLE (bottom) in the F435W, F606W, and F814W filters (from left to right). The scale of the original and ICL+background images is the same for each filter. 


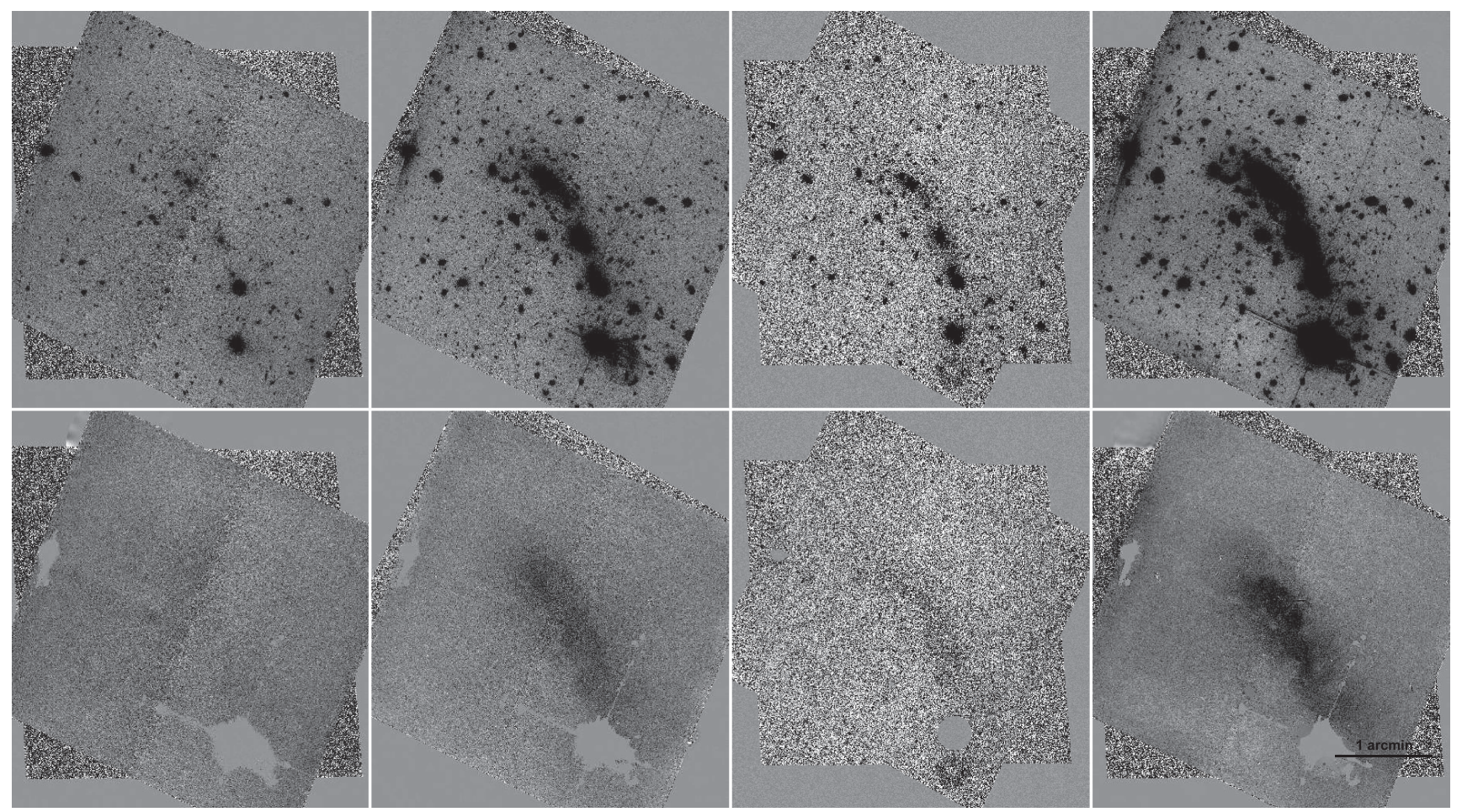

Figure 10. Original images of the unrelaxed cluster MACS 0416 (top) and ICL+background maps provided by CICLE (bottom) in the F435W, F606W, F625W, and F814W filters (from left to right). The scale of the original and ICL+background images is the same for each filter.

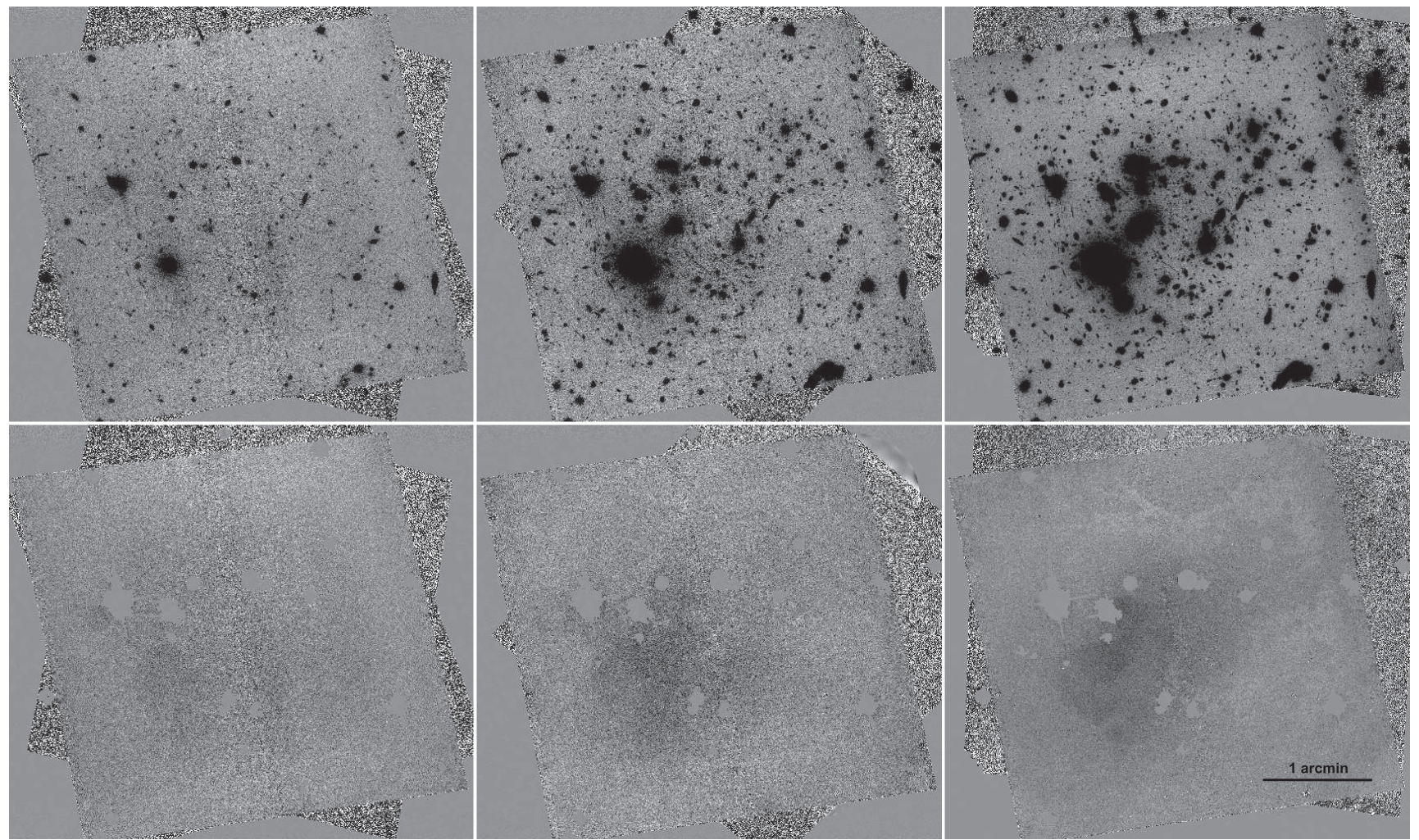

Figure 11. Original images of the unrelaxed cluster MACS 0717 (top) and ICL+background maps provided by CICLE (bottom) in the F435W, F606W, and F814W filters (from left to right). The scale of the original and ICL+background images is the same for each filter. 

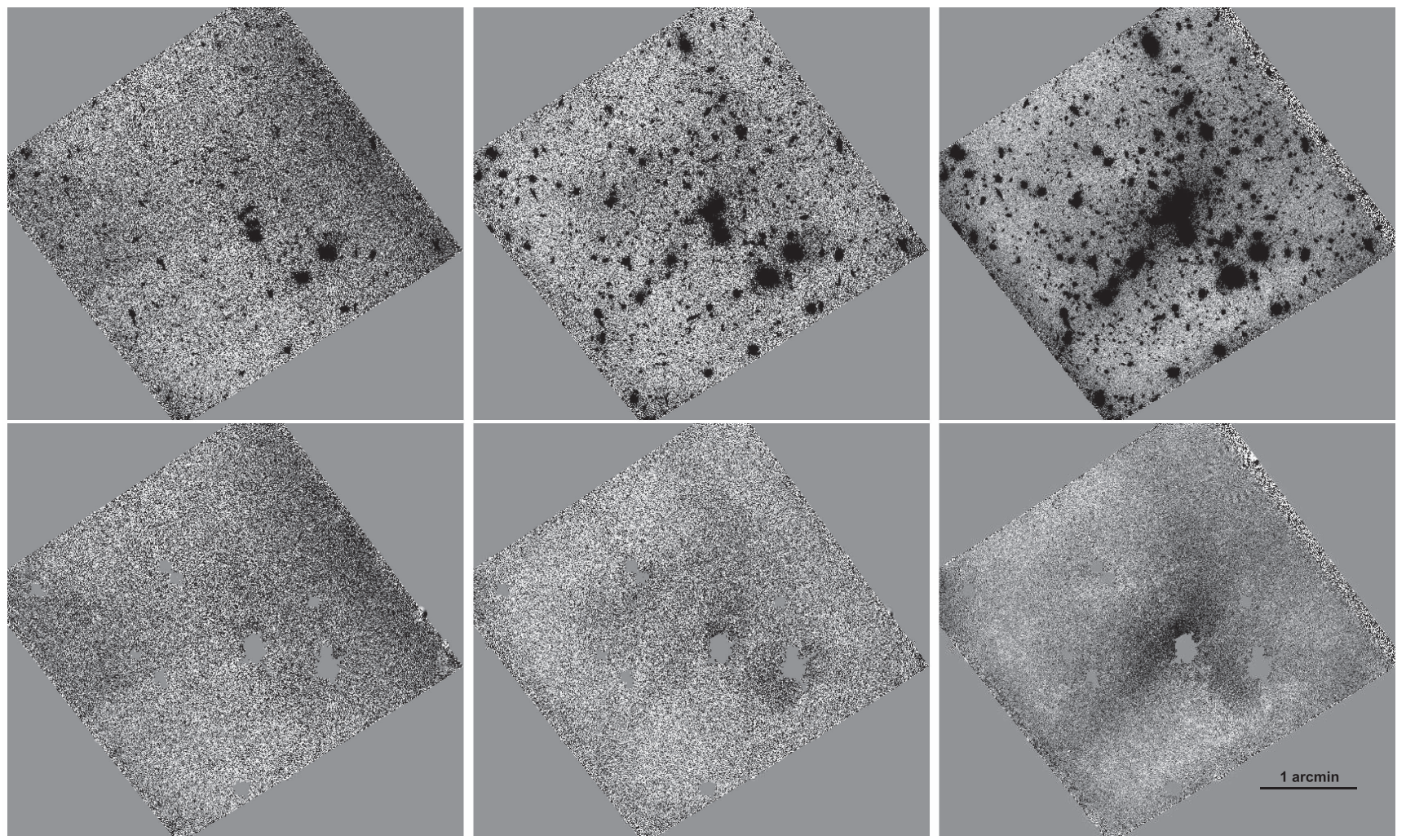

Figure 12. Original images of the unrelaxed cluster MACS 1149 (top) and ICL+background maps provided by CICLE (bottom) in the F435W, F606W, and F814W filters (from left to right). The scale of the original and ICL+background images is the same for each filter.
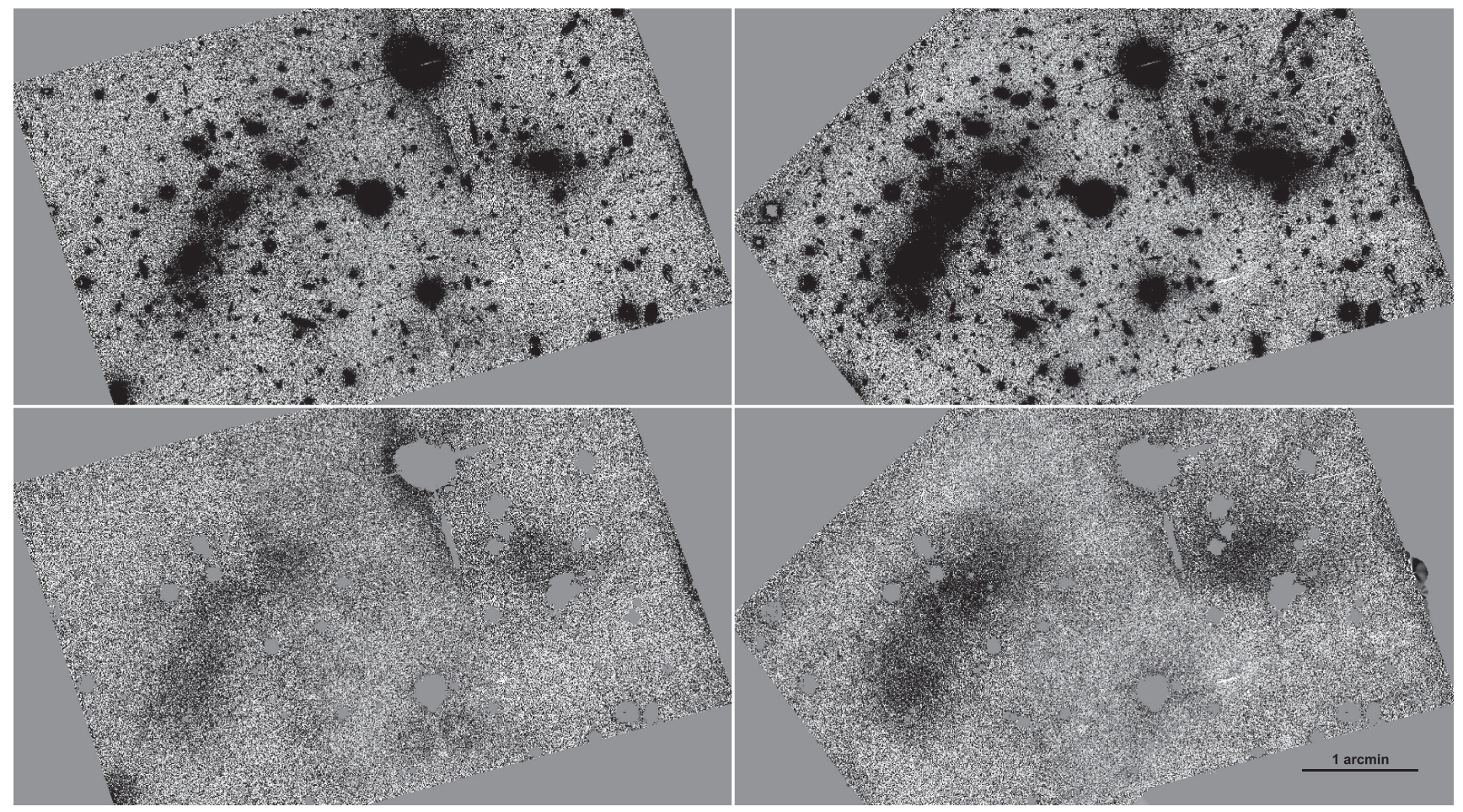

Figure 13. Original images of the unrelaxed Bullet cluster (top) and ICL+background maps provided by CICLE (bottom) in the F606W and F814W filters (from left to right). The scale of the original and ICL+background images is the same for each filter. 


\section{ORCID iDs}

Yolanda Jiménez-Teja (1) https://orcid.org/0000-00026090-2853

Anton M. Koekemoer (iD https://orcid.org/0000-00026610-2048

Adi Zitrin (iD https://orcid.org/0000-0002-0350-4488

Keiichi Umetsu (iD https://orcid.org/0000-0002-7196-4822

Holland Ford (iD https://orcid.org/0000-0001-8505-9535

Rychard J. Bouwens (i) https://orcid.org/0000-0002-

4989-2471

Larry D. Bradley (iD https://orcid.org/0000-0002-7908-9284

Dan Coe (iD https://orcid.org/0000-0001-7410-7669

Megan Donahue (1) https://orcid.org/0000-0002-2808-0853

Leopoldo Infante (iD https://orcid.org/0000-0001-8581-932X

Daniel D. Kelson (iD https://orcid.org/0000-0003-4727-4327

Elinor Medezinski ii https://orcid.org/0000-0001-7007-2358

Peter Melchior (iD https://orcid.org/0000-0002-8873-5065

Massimo Meneghetti (iD https://orcid.org/0000-0003-

1225-7084

Alberto Molino (iD https://orcid.org/0000-0002-5007-680X

Leonidas A. Moustakas (ib https://orcid.org/0000-0003-

3030-2360

Jack Sayers (ib https://orcid.org/0000-0002-8213-3784

Stella Seitz (ib https://orcid.org/0000-0003-3277-2243

Wei Zheng (iD https://orcid.org/0000-0002-0205-5174

\section{References}

Adami, C., Durret, F., Guennou, L., \& Da Rocha, C. 2013, A\&A, 551, A20 Adami, C., Slezak, E., Durret, F., et al. 2004, A\&A, 429, 39

Balestra, I., Mercurio, A., Sartoris, B., et al. 2016, ApJS, 224, 33

Barrena, R., Biviano, A., Ramella, M., Falco, E. E., \& Seitz, S. 2002, A\&A, 386, 816

Bernstein, G. M., Nichol, R. C., Tyson, J. A., et al. 1995, AJ, 110, 1507

Bertin, E., \& Arnouts, S. 1996, A\&AS, 117, 393

Bonafede, A., Brüggen, M., van Weeren, R., et al. 2012, MNRAS, 426, 40

Boschin, W., Girardi, M., Spolaor, M., \& Barrena, R. 2006, A\&A, 449, 461

Boyd, J. P. 2000, Chebyshev and Fourier Spectral Methods (2nd ed.; New York: Dover)

Bravi, L., Gitti, M., \& Brunetti, G. 2016, MNRAS, 455, L41

Brunetti, G., \& Jones, T. W. 2014, IJMPD, 23, 1430007

Burke, C., Collins, C. A., Stott, J. P., \& Hilton, M. 2012, MNRAS, 425, 2058

Burke, C., Hilton, M., \& Collins, C. A. 2015, MNRAS, 449, 2353

Cassano, R., Brunetti, G., Giocoli, C., \& Ettori, S. 2016, A\&A, 593, A81

Cassano, R., Ettori, S., Giacintucci, S., et al. 2010, ApJL, 721, L82

Connor, T., Donahue, M., Kelson, D. D., et al. 2017, ApJ, 848, 37

Contini, E., De Lucia, G., Villalobos, Á., \& Borgani, S. 2014, MNRAS, 437, 3787

Couch, W. J. 1998, ApJ, 497, 188

Couch, W. J., \& Sharples, R. M. 1987, MNRAS, 229, 423

Cuciti, V., Cassano, R., Brunetti, G., et al. 2015, A\&A, 580, A97

Da Rocha, C., \& Mendes de Oliveira, C. 2005, MNRAS, 364, 1069

Da Rocha, C., Ziegler, B. L., \& Mendes de Oliveira, C. 2008, MNRAS, 388, 1433

DeMaio, T., Gonzalez, A. H., Zabludoff, A., Zaritsky, D., \& Bradač, M. 2015, MNRAS, 448, 1162

DeMaio, T., Gonzalez, A. H., Zabludoff, A., Zaritsky, D., \& Connor, T. 2018, MNRAS, 474, 3009

Donahue, M., Ettori, S., Rasia, E., et al. 2016, ApJ, 819, 36

Ebeling, H., Ma, C-J., \& Barrett, E. 2014, ApJS, 211, 21

Enßlin, T. A., Biermann, P. L., Klein, U., \& Kohle, S. 1998, A\&A, 332, 395
Fadda, D., Girardi, M., Giuricin, G., Mardirossian, F., \& Mezzetti, M. 1996, ApJ, 473, 670

Feldmeier, J., Mihos, J. C., Morrison, H. L., Rodney, S. A., \& Harding, P. 2002, ApJ, 575, 779

Giacintucci, S., Markevitch, M., Cassano, R., et al. 2017, ApJ, 841, 71

Giovannini, G., Tordi, M., \& Feretti, L. 1999, NewA, 4, 141

Girardi, M., Fadda, D., Giuricin, G., Mardirossian, F., \& Mezzetti, M. 1996, ApJ, 457, 61

Goddard, D., Thomas, D., Maraston, C., et al. 2016a, MNRAS, 465, 688

Goddard, D., Thomas, D., Maraston, C., et al. 2017b, MNRAS, 466, 4731

Golovich, N., Dawson, W. A., Wittman, D., et al. 2016, ApJ, 831, 110

Gonzaga, S., Hack, W., Fruchter, A., et al. 2012, The DrizzlePac Handbook (Baltimore, MD: STScI)

Govoni, F., Enßlin, T. A., Feretti, L., \& Giovannini, G. 2001a, A\&A, 369, 441

Govoni, F., Feretti, L., Giovannini, G., et al. 2001b, A\&A, 376, 803

Guennou, L., Adami, C., Da Rocha, C., et al. 2012, A\&A, 537, A64

Holwerda, B. W. 2005, arXiV:astro-ph/0512139

Jiménez-Teja, Y., \& Benítez, N. 2012, ApJ, 745, 150

Jiménez-Teja, Y., \& Dupke, R. 2016, ApJ, 820, 49

Kale, R., Venturi, T., Giacintucci, S., et al. 2013, A\&A, 557, A99

Kale, R., Venturi, T., Giacintucci, S., et al. 2015, A\&A, 579, A92

Kempner, J. C., \& David, L. P. 2004, MNRAS, 349, 385

Koekemoer, A. M., Faber, S. M., Ferguson, H. C., et al. 2011, ApJS, 197, 36

Koekemoer, A. M., Fruchter, A. S., Hook, R. N., \& Hack, W. 2002, in HST Calibration Workshop, ed. S. Arribas, A. Koekemoer, \& B. Whitmore (Baltimore: STScI), 337

Krick, J. E., \& Bernstein, R. A. 2007, AJ, 134, 466

Liang, H., Hunstead, R. W., Birkinshaw, M., \& Andreani, P. 2000, ApJ, 544,686

Livermore, R. C., Finkelstein, S. L., \& Lotz, J. M. 2017, ApJ, 835, 113

Lotz, J. M., Koekemoer, A., Coe, D., et al. 2017, ApJ, 837, 97

Maier, C., Kuchner, U., Ziegler, B. L., et al. 2016, A\&A, 590, A108

Markevitch, M., Gonzalez, A. H., Clowe, D., et al. 2004, ApJ, 606, 819

Markevitch, M., Gonzalez, A. H., David, L., et al. 2002, ApJL, 567, L27

Martinet, N., Durret, F., Adami, C., \& Rudnick, G. 2017, A\&A, 604, A80

Mason, J. C., \& Handscomb, D. 2003, Chebyshev Polynomials (Boca Raton, FL: CRC Press)

Mercurio, A., Girardi, M., Boschin, W., et al. 2003a, A\&A, 397, 431

Mercurio, A., Massarotti, M., Merluzzi, P, et al. 2003b, A\&A, 408, 57

Merlin, E., Amorin, R., Castellano, M., et al. 2016, A\&A, 590, A30

Merritt, D. 1984, ApJ, 276, 26

Merten, J., Coe, D., Dupke, R., et al. 2011, MNRAS, 417, 333

Merten, J., Meneghetti, M, Postman, M., et al. 2015, ApJ, 806, 4

Molino, A., Benítez, N., Ascaso, B., et al. 2017, MNRAS, 470, 95

Montes, M., \& Trujillo, I. 2014, ApJ, 794, 137

Montes, M., \& Trujillo, I. 2018, MNRAS, 474, 917

Morishita, T., Abramson, L. E., Treu, T., et al. 2017, ApJ, 846, 139

Murante, G., Giovalli, M., Gerhard, O., et al. 2007, MNRAS, 377, 2

Ogrean, G. A., van Weeren, R. J., Jones, C., et al. 2016, ApJ, 819, 113

Owers, M. S., Randall, S. W., Nulsen, P. E. J., et al. 2011, ApJ, 728, 27

Pandey-Pommier, M., Richard, J., Combes, F., et al. 2016, in Proc. Annual Meeting of the French Society of Astronomy and Astrophysics, SF2A2016, ed. C. Reylé et al. (Paris: SF2A), 367

Pierini, D., Zibetti, S, Braglia, F., et al. 2008, A\&A, 483, 727

Postman, M., Coe, D., Benítez, N., et al. 2012, ApJS, 199, 25

Presotto, V., Girardi, M., Nonino, M., et al. 2014, A\&A, 565, A126

Rudick, C. S., Mihos, J. C., \& McBride, C. K. 2006, ApJ, 648, 936

Rudick, C. S., Mihos, J. C., \& McBride, C. K. 2011, ApJ, 732, 48

Shimwell, T. W., Brown, S., Feain, I. J., et al. 2014, MNRAS, 440, 2901

Springel, V., \& Farrar, G. R. 2007, MNRAS, 380, 911

Umetsu, K., Medezinski, E., Nonino, M., et al. 2014, ApJ, 795, 163

Van Weeren, R. J., Röttgering, H. J. A., Brüggen, M., \& Cohen, A. 2009, A\&A, 505, 991

Venturi, T., Giacintucci, S., Brunetti, G., et al. 2007, A\&A, 463, 937

Venturi, T., Giacintucci, S., Dallacasa, D., et al. 2008, A\&A, 484, 327

Willman, B., Governato, F., Wadsley, J., \& Quinn, T. 2004, MNRAS, 355, 159 